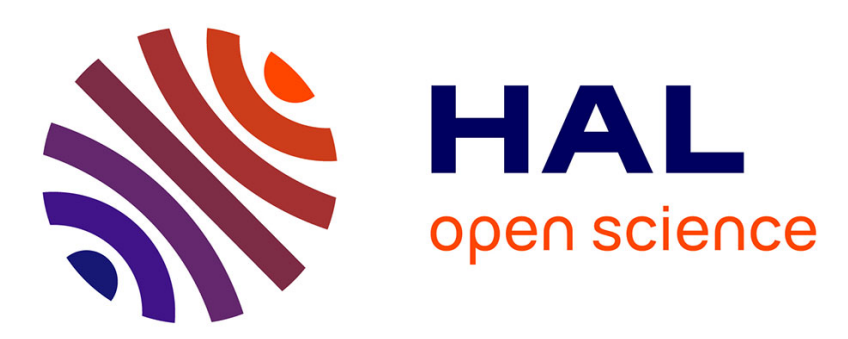

\title{
Rich Spray-Flame Propagating through a 2D-Lattice of Alkane Droplets in Air
}

Colette Nicoli, Bruno Denet, Pierre Haldenwang

\section{To cite this version:}

Colette Nicoli, Bruno Denet, Pierre Haldenwang. Rich Spray-Flame Propagating through a 2D-Lattice of Alkane Droplets in Air. Combustion and Flame, 2015, 162 (12), pp.4598-4611. 10.1016/j.combustflame.2015.09.018 . hal-01255816

\section{HAL Id: hal-01255816 https://hal.science/hal-01255816}

Submitted on 14 Jan 2016

HAL is a multi-disciplinary open access archive for the deposit and dissemination of scientific research documents, whether they are published or not. The documents may come from teaching and research institutions in France or abroad, or from public or private research centers.
L'archive ouverte pluridisciplinaire HAL, est destinée au dépôt et à la diffusion de documents scientifiques de niveau recherche, publiés ou non, émanant des établissements d'enseignement et de recherche français ou étrangers, des laboratoires publics ou privés. 


\title{
Rich Spray-Flame Propagating
}

\section{through a 2D-Lattice of Alkane Droplets in Air}

\author{
C. Nicoli ${ }^{1}$, B. Denet ${ }^{2}$, P. Haldenwang ${ }^{1}$ \\ ${ }^{1}$ M2P2 , Aix-Marseille Université/ CNRS/ Centrale Marseille, UMR 7340, 13451 Marseille France, \\ ${ }^{2}$ IRPHE, Aix-Marseille Université/ CNRS/ Centrale Marseille, UMR 7342, 13384 Marseille France
}

\begin{abstract}
In a recent numerical paper (Nicoli et al. Combust. Sci. Technol. vol. 186, pp. 103-119; 2014) [1], a model of isobaric flame propagation in lean sprays has been proposed. The initial state of the monodisperse mists was schematized by a system of individual alkane droplets initially located at the nodes of a facecentered 2D-lattice, surrounded by a saturated mixture of alkane and air. In the present study, the previous model is complemented with an original chemical scheme that allows us to study the combustion of rich alkane/air mixtures.

The main parameters of this configuration are $S$, the lattice spacing (in reactive-diffusive length units), $\varphi_{L}$, the liquid loading (or equivalence ratio relative to the fuel under liquid phase), and $\varphi_{G}$ (with $\varphi_{G} \leq 0.8$ ), the gaseous equivalence ratio (i.e. that corresponding to the saturated vapour pressure in the fresh spray). We presently focus on sprays, the overall equivalence ratio of which is within the range $1 \leq\left(\varphi_{L}+\varphi_{G}\right) \leq 1.85$.

For a large set of parameters, we retrieve a feature often observed on the rich side in the experiments: flame propagation in the presence of droplets can be faster than the pure premixed flames with the same overall equivalence ratio. This is mainly observed when the lattice spacing is sufficiently large. However, the study underlines the role played by the velocities of two particular single-phase premixed flames: the "initial vapour flame" that only burns (if any) the mixture due to the saturated vapour and the "all fuel flame" that propagates (if any) in a mixture where all fuel is vaporized and mixed. When the "initial vapour flame" is too slow (i.e. a feeble spray Peclet number), the spray-flame speed results from the competition between two mechanisms: a speed chemically enhanced due to some enrichment coming from vaporization (possibly bounded by the "all fuel flame" speed) and a slowing down in flame velocity because the vaporization time scale sets the pace on combustion. On the other hand, for large spray Peclet number, the upper flammability limit is found to be strongly enlarged, and the spray-flame propagates with the velocity of the "initial vapour flame".

Moreover, the flame structure deeply depends on lattice spacing: for a large lattice, the combustion stage mainly corresponds to a triple flame, with the diffusion flame that develops around the oxygen pocket located behind the lean wing of the flame front (i.e. far from the droplets). On the other hand, as $s$ decreases, this diffusion flame tends to be more and more incorporated into the flame front.
\end{abstract}

Keywords : spray-flame; two-phase combustion; heterogeneous combustion; stratified combustion; droplet array combustion, flamelet model. 


\section{Nomenclature}

$D_{t h} \quad$ thermal diffusivity

$F\left(\varphi_{u}\right)$ heat of reaction depending on unburnt mixture equivalence ratio

$I_{W}^{-} \quad$ marker of diffusion flame (i.e. the negative part of the indexed reaction rate)

$L \quad$ latent heat of fuel vaporisation

$L e_{i} \quad$ Lewis number of species $i$ in the mixture

$P \quad$ pressure

$p_{F} \quad$ partial pressure of fuel

$P e_{s} \quad$ spray Peclet number

$Q^{*} \quad$ effective heat of reaction at stoichiometry

$R \quad$ droplet radius

$S \quad$ lattice spacing

$T_{A} \quad$ activation temperature

$T_{b}^{*} \quad$ adiabatic flame temperature for stoichiometric gaseous mixture

$T_{u} \quad$ temperature of the fresh gaseous mixture

$U_{L}(\varphi)$ adiabatic flame speed for the single-phase premixture of equivalence ratio $\varphi$

$U_{L}^{*}=U_{L}(\varphi=1)$ adiabatic flame speed for the stoichiometric single-phase premixture

$W \quad$ reaction rate

$Y_{i} \quad$ mass fraction of species $i$ in the mixture

$Z_{F} \quad$ fuel mixture fraction (that follows the fuel atoms)

$Z_{O} \quad$ oxygen mixture fraction (that follows the oxidizing atoms)

$\mathrm{Ze} \quad$ Zeldovich number for stoichiometric gaseous mixture

$\delta_{L}^{*} \quad$ adiabatic flame thickness for the stoichiometric gas mixture

$\varphi \quad$ equivalence ratio

$\varphi_{u} \quad$ local estimate of the unburnt mixture equivalence ratio

$\varphi_{L} \quad$ liquid equivalence ratio of the fresh spray (or liquid loading)

$\varphi_{G} \quad$ (initial) gaseous equivalence ratio of the fresh spray (a function of the saturated vapour pressure)

$\varphi_{T} \quad$ overall equivalence ratio of the fresh spray

$\lambda(T) \quad$ thermal conductivity (here, a function of temperature)

$\psi_{i} \quad$ reduced mass fraction of specie $i$

$\rho \quad$ density

$\theta \quad$ reduced temperature 


\begin{abstract}
“*” (resp. “^”) superscript indicates the value at stoichiometric (resp. saturated vapour) conditions "u" (resp. "b") subscript indicates the value for fresh (resp. burnt) mixture "L" (resp. "G") subscript indicates the value for liquid (resp. gaseous) phase
\end{abstract}

\title{
1. Introduction
}

Combustion spreading through a spray concerns a large number of applications, such as Diesel engines or rocket engines, gas turbines or industrial furnaces. In a recent study [1], it has been performed numerical simulations of isobaric flames propagating through a face-centred 2D-lattice of droplets. The lattice was conceived as a schematization of a particular initial state of the fresh spray. Since the purpose was the lean sprays, it has been observed that the results very weakly depended on the chemical scheme: the classical onestep irreversible chemistry with a global exothermic reaction was found to be sufficient for exhibiting the main features of the combustion spread in a lean mist.

Contrarily to that previous work on lean sprays, we are now interested in rich spray combustion. In [2], we have shown that the results of numerical simulations in rich sprays can strongly depend on the selected chemical scheme. Although the overall spray composition is supposed to be rich, the local equivalence ratio can be found lean far from the droplets, if the saturated vapour pressure of fuel is low. To study the combustion in such a heterogeneous medium, we have implemented a chemical scheme, which is a composition-corrected one-step global reaction. To obtain satisfactory properties we have proposed to adapt the heat release to the equivalence ratio of the fresh gaseous mixture, that results from droplet vaporization and the subsequent mixture of fuel with air. This chemical scheme introduces two progress variables that allow us to adapt heat release to fresh composition. This procedure gives satisfaction on both lean and rich sides [2].

The problem of flame speed enhancement by droplets has a long history; an interesting summary of the early works has been carried out by Myers and Lefebvre (1986) [3]. Let us particularly quote the works by Cekalin (1961) [4] and by Mizutani and Nakajima (1973) [5-a, 5-b], who added kerosene droplets to a propane air mixture and saw an increase in propagation speed. We also have to mention the pioneering works of Hayashi and Kumagai (1975) [6] and Hayashi et al. (1976) [7], who used a Wilson cloud chamber to produce a nearly monodisperse spray. For polydisperse kerosene sprays, Polymeropoulos and Das (1975) [8] observed that burning velocity reaches a maximum for a certain domain of droplet size.

The situation concerning the velocity increase is, however, not completely clear. For ethanol and isooctane sprays, Hayashi and Kumagai (1975) [6] and Hayashi et al. (1976) [7] reported velocity enhancement for rich sprays, and for lean sprays with large droplets. But, Ballal and Lefebvre (1981) [9] for isooctane, and Myers and Lefebvre (1986) [3] with six different fuels, did not observe the enhancement effect for lean sprays. Our recent numerical study on flames propagating in a lean droplet lattice [1] tends to confirm the latter observations: no increase in spray-flame velocity were noticeable for lean alkane sprays. There are nevertheless other experiments reported in the literature where a velocity increase occurs: for instance, in the lean spray case, a more recent experimental study by Nomura et al. (2000) [10] on ethanol sprays in microgravity indicates larger propagation speeds in a spray than in the equivalent premixed flame, when the droplet size belongs to some interval. 
Because it focuses on the droplets, our small scale DNS study does not contain an important effect observed in the experiments: the role of the flame front instabilities (see, for instance, the recent works by Bradley et al. (2014) [11] and by Nassouri et al. (2013) [12] in the case of the expanding spherical sprayflames). Given the importance of these effects in numerous experiments, we now discuss this question more deeply.

Various experiments have revealed that flame spreading in a rich spray has a propagation velocity larger than that of the equivalent premixed flame. In a number of these experiments, the flame front was found corrugated, with a large number of cells. Therefore, spray-flame speed enhancement could possibly be explained by instabilities of the front. As a matter of fact, the interplay between instabilities and droplets seems to have a peculiar importance for the spherical flames, a case that has extensively been studied in the recent years [11-13]: due to gas expansion and droplet inertia, front acceleration modifies vaporization which in turn modifies combustion spreading. Moreover, even for the pure gaseous flame, we know that the spherical flame is subject to highly non-linear effects, leading to the creation of a lot of cells and even to an acceleration of the flame speed (called self-acceleration), see the experiments by Bradley et al. (2001) [14]. In this regard, the experiments with droplets lead more or less to the same effects, except that the inhomogeneity seen by the flame front is here caused by droplets, and not by turbulence.

On the theoretical side, similar conclusions can be drawn. The creation of many cells in spherical premixed flames (without droplets) has long been modeled in the case of the hydrodynamic instability with the Sivashinsky equation, namely in 2D by Karlin and Sivashinsky (2007) [15], Fursenko et al. (2008) [16], (see also the simulations of D'Angelo et al. (2000) [17] in the 3D case, which could be compared to the experiments with droplets). This model equation contains the two main effects, creation of many cells and self-acceleration. In the related case of the Sivashinsky equation close to a parabolic shape, it has been shown in Denet and Joulin (2011) [18] that, as the stretch is reduced (as the front is less and less curved), solutions with a lot of cells appear, the same effect as the one observed in the spherical configuration. Every theoretical approach stresses on the major role played by high-level noise, which can here be triggered by the droplets.

A droplet lattice, as depicted by the left (unburnt) parts of the various fields drawn in Fig.1, is a manner of controlling the spray initial conditions. Other attempts exist in the literature. For instance, Mikami et al. (2006) [19] measured the flame spread along an array of anchored n-decane droplets. In this microgravity experiment, droplet size and transverse interdroplet distance were fixed, only the interdroplet distance in the direction of spreading was changed. The purpose was to investigate different modes of droplet combustion, from individual to group combustion. The domain of overall equivalence ratio considered in [19] is much higher than the one studied here.

In what concerns the recent spherical spray-flames as studied in [11-12], we would warn the Reader that such experiments cumulate many physical effects, and consequently are not easy to interpret. For instance, the self-acceleration of flame is observed in [11], but the effect of instabilities seems to be limited to a relatively mild increase of $50 \%$ in the reported flame velocity; it could be more in other experiments, particularly at high pressure. Our present DNS study being performed in a small domain to focus on dropletflame interplay, we hence do not pretend to reproduce the features observed in the above-mentioned experiments, especially the front instabilities.

Let us finally note that the present DNS -as described in detail below- solves the vaporization and the combustion of "thick" individual droplets. This is in contrast to simulations that use point droplets, such as all 
1D numerical studies on spray-flame, as in Aggarwal and Sirignano (1985) [20], Silverman et al. (1993) [21], Suard, et al. (2004) [22] and Neophytou and Mastorakos (2009) [23], or in the 2D analysis by Reveillon and Vervisch (2005) [24]. By contrast, the present DNS fully resolves the droplets as in the numerical approach by Kikuchi et al. (2005) [25]; here, we additionally allow the droplets to be moved by the gas expansion.

The paper is structured as follows. First, the spray-flame modelling is presented, the chemical scheme being briefly described. Second, the flame spread through the lattice of droplets is numerically studied. The spray-flame speed is defined, and the flame structure is discussed. Finally, the role played by the various parameters is described.

\section{Heterogeneous modelling of spray flames}

At low pressure, flame thickness in standard sprays appears large in comparison with droplet interspacing. In the recent years, this property allowed us to resort to a homogenization process for developing an appropriate numerical modelling [22, 26-27]. In such an approach which also neglects droplet inertia, liquid fuel appears as an additional species only allowed to enter into the chemical scheme after a vaporization step. Several spreading regimes have been predicted [22], in particular an intrinsic oscillatory regime, which had been observed experimentally by Hanai et al. [28] and by Atzler [13]. The existence of this regime, which occurs as a Hopf bifurcation, does not require the presence of differential diffusivity effects [26-27].

At moderate and high pressures, spray-flame thickness can no longer be large enough -in comparison with droplet interspacing- to allow any process of homogenization. In such a system where both phases are initially in equilibrium, the spray-flame tends to be controlled by vaporization, the chemical heat release permitting the vaporization of the droplets one after another. Therefore, the spray-flame propagates within a heterogeneous (or stratified) mixture with droplets. The mist initial structure is represented as a face-centred 2D-lattice of alkane droplets in a pre-mixture of alkane-air (see Fig.1). Then, the droplets can move as the flame propagates.

This work is hence devoted to spray with droplet inter-distance not small in comparison with the characteristic combustion scales (at least, of the same order). Our numerical modelling starts from the usual set of conservation laws: mass, momenta, energy and species. The non-dimensional form of the conservation laws is performed with the use of the units related the stoichiometric (gaseous) premixed flame, flame thickness, flame velocity, as already used in [1]. A brief description of the overall approach is provided in Appendix A.

\subsection{General considerations}

Since the accurate chemical schemes for alkane are too complex for efficient numerical simulations, a simplified chemical kinetics is generally recommended. It is long known that the classical one-step Arrhenius law (equipped with constant heat release) largely overestimates the adiabatic flame temperature on the rich side. To overcome the difficulty to assess the main rich flame characteristics (as speed and temperature), we have considered [29] a simple modification of the one-step chemical scheme: heat release becomes a function, denoted by $F\left(\varphi_{u}\right)$, of the fresh mixture equivalence ratio $\varphi_{u}$ (see below the definition of $\varphi_{u}$ ). In practice, this model is able to correctly mimic the premixed single-phase flame characteristics (adiabatic 
flame temperature and flame speed). As a matter of fact, this adaptation accounts for all the species in equilibrium at the actual flame temperature. This approach of flamelet type requires to know -for any point within the computational domain- the actual composition of the corresponding fresh premixture, already denoted $\varphi_{u}$. Section 2 is mainly devoted to the description of this flamelet approach.

Let us first introduce the irreversible single-step reaction for alkane-air combustion

$$
\mathrm{C}_{n} \mathrm{H}_{2 n+2}+\left(\frac{3 n+1}{2}\right)\left[\mathrm{O}_{2}+\frac{v_{N}}{v_{O}} N_{2}\right] \rightarrow n \mathrm{CO}_{2}+(n+1) \mathrm{H}_{2} \mathrm{O}+\left(\frac{3 n+1}{2}\right) \frac{v_{N}}{v_{O}} N_{2}
$$

This single reaction allows us to characterize the local mixture composition by $\varphi$, the field of equivalence ratio, which reads

$$
\varphi=\frac{Y_{F}}{Y_{O}} \times \frac{v_{O} M_{O}}{v_{F} M_{F}}
$$

where $v_{i}$ stands for the stoichiometric coefficient of species $i, M_{i}$, the molar mass of species $i$. In fresh air, $v_{N} / v_{O}=3.76$ is the molar ratio of nitrogen to oxygen, while the mass ratio is $v_{N / O}=v_{N} M_{N} / v_{O} M_{O}=3.29$. At stoichiometry, let us denote by $Q^{*}$, the combustion heat related to the flame temperature (i.e. $T_{b}^{*}$ ), in accordance with

$$
T_{b}^{*}=T_{u}+\frac{\left(Y_{F}\right)_{u}^{*} Q^{*}}{C_{p} v_{F} M_{F}}
$$

We denote by $U_{L}^{*}$, the laminar (single-phase) flame speed, that characterizes the combustion of a premixed fresh mixture of unity equivalence ratio (i.e. $\varphi=1$ ). In what follows, the subscript "u" [resp. "b"] is associated with gaseous fresh [resp. burnt] mixture. Thus, $D_{t h, b}^{*}$ denotes the thermal diffusivity coefficient of the burnt gases in the stoichiometric (gaseous) flame. The non-dimensioning process described in Appendix A uses $l_{R D}^{*}=D_{t h, b}^{*} / U_{L}^{*}$ and $\tau_{R D}^{*}=D_{t h, b}^{*} /\left(U_{L}^{*}\right)^{2}$ as length and time scales, respectively.

Furthermore, we handle temperature and species mass fractions under the reduced forms

$$
\theta=\left(T-T_{u}\right) /\left(T_{b}^{*}-T_{u}\right) ; \quad \psi_{i}=Y_{i} / Y_{i, u}^{*}
$$

( $i=f$ for the alkane fuel, $i=o$ for oxygen and $i=p$ for combustion products)

Appendix A recalls the system (A.14-A.17) of governing equations that are derived from the general laws of conservation. Equation (A.14) deserves a particular attention

$$
\frac{\partial \theta}{\partial t}+\bar{V} \cdot \bar{\nabla} \theta=\frac{1}{\rho} \operatorname{div}\left(\rho D_{t h} \bar{\nabla} \theta\right)+F\left(\varphi_{u}\right) W\left(\rho, \psi_{i}, \theta\right)
$$

where $W$, the reaction rate, is defined in (A.11), and $D_{t h}$ is a strongly non-linear function of temperature, which prohibits the diffusion phenomena at low temperature. Vector $\bar{V}$ stands for the velocity field that is governed by the Navier-Stokes equations (A.16-A.17). 
In Equ. (5), appears $F\left(\varphi_{u}\right)$, a quantity adjustable to the fresh mixture composition $\varphi_{u}$, which will allow us to tackle the problem of flame propagation in stratified medium. Let us first propose the following definitions.

The (initial) overall equivalence ratio of the spray is given by the normalized ratio of the total amount of fuel to the total amount of oxygen in the lattice

$$
\varphi_{T}=\frac{v_{O} M_{O}}{v_{F} M_{F}} \frac{\int_{\text {initial latice }} \rho_{F} d v}{\int_{\text {inital lattice }} \rho_{O} d v},
$$

while the quantity $\varphi_{L}$ [resp. $\varphi_{G}$ ] takes account of the fuel density under liquid phase [resp. gas phase], only. We obviously have $\varphi_{T}=\varphi_{L}+\varphi_{G}$. In the present study, the range selected for $\varphi_{T}$ is limited to $1 \leq \varphi_{T} \leq 1.85$. In Appendix $\mathrm{B}$, it is show how $\varphi_{G}$ (or the saturated vapour pressure) can in practice be controlled without affecting the scalings of the paper.

Before combustion reaches a certain locus of the fresh spray, the local equivalence ratio $\varphi_{u}$ is an unsteady quantity since it depends on vaporization and mass transfer: it can vary from $\varphi_{G}$ (far from the droplets) to a very high value (nearly pure fuel close to the droplets). Two particular single-phase flames can be considered in this context. On the one hand, if all fuel is mixed before burning, we can be faced with a rich premixed flame, the laminar velocity of which is denoted by $U_{L}\left(\varphi_{T}\right)$. On the other hand, if the initial saturated fuel vapour (mixed with air) is flammable, a combustion spreading can occur rapidly, so that the fuel in the droplets has no time to vaporize. In this case, we are concerned with the propagation of a premixed flame, the laminar velocity of which is $U_{L}\left(\varphi_{G}\right)$. To discuss the importance of the latter situation, let us now consider $\tau_{\text {vap }}$, the characteristic time to vaporize (and mix) the droplets. Supposing that $\tau_{\text {vap }}$ follows the "D2law", we obtain $\tau_{\text {vap }} \square \rho_{L} / \rho_{G} \times(2 R)^{2} / D_{\text {th }}$. Furthermore, we introduce $\tau_{\text {prop }}$, the characteristic time of propagation in the lattice (without resorting to the liquid fuel), which behaves like $\tau_{\text {prop }} \square s / U_{L}\left(\varphi_{G}\right)$. The comparison of both characteristic times invites us to define $P e_{s}$, the Peclet number of spray combustion, as

$$
P e_{s}=\frac{\rho_{L}}{\rho_{G}} \frac{(2 R)^{2}}{D_{t h, b}^{*}} \times \frac{U_{L}\left(\varphi_{G}\right)}{s}
$$

where $\rho_{L}, \rho_{G}, R$ and $s$ are respectively the density of the liquid, the density of the gas, the droplet radius and the lattice spacing. In the case of large $P e_{s}$, we expect that $U_{L}\left(\varphi_{G}\right)$ will provide us with the spray-flame speed.

\subsection{Flamelets in stratified medium}

When the assumptions $D_{F} \approx D_{O_{2}} \approx D_{P}$ hold for the diffusivities of fuel, oxygen and products, the literature on premixed flames considers that certain combinations of variables are preserved from the fresh 
gases to the burnt gases. In the present work, we select two mixture variables which involve either fuel and products mass fractions, or oxidant and products mass fractions, namely:

$$
Z_{F}=\frac{Y_{F}}{v_{F} M_{F}}-\frac{Y_{P}}{v_{P} M_{P}} \quad \text { and } \quad Z_{O}=\frac{Y_{O_{2}}}{v_{O_{2}} M_{O_{2}}}-\frac{Y_{P}}{v_{P} M_{P}}
$$

Note that $v_{p}$ is a negative coefficient in equations (8.a-b) and (A.2) for $i=p$. Consequently, under the above assumptions of equal diffusivity of the species, the $Z_{F}$ and $Z_{O}$ fields are obviously governed by the nonreactive equation

$$
\frac{\partial Z}{\partial t}+\bar{V} \cdot \bar{\nabla} Z=\frac{1}{\rho} \operatorname{div}(\rho D \bar{\nabla} Z)
$$

which results from combinations of equations (A.2).

Hence, at the flame characteristic length scale, which is supposed much smaller that the droplet interspacing, $Z_{F}$ and $Z_{O}$ can be considered as conserved when crossing the flame front. Since $Z_{F}$ and $Z_{O}$ are conserved through the flame, their ratio $Z_{F} / Z_{O}$ is also conserved.

$$
Z_{F} / Z_{O}=\left\{\left[Y_{F}-\left(v_{F} M_{F} / v_{P} M_{P}\right) Y_{P}\right] /\left[Y_{O_{2}}-\left(v_{O_{2}} M_{O_{2}} / v_{P} M_{P}\right) Y_{P}\right]\right\} \times\left\{v_{O_{2}} M_{O_{2}} / v_{F} M_{F}\right\}
$$

where $Y_{P}=1-Y_{F}-Y_{O}-Y_{N}$ and $Y_{N}=\left(v_{N_{2}} M_{N_{2}}\right) /\left(\varphi_{T} v_{F} M_{F}+v_{O_{2}} M_{O_{2}}+v_{N_{2}} M_{N_{2}}\right)$

Now, in the fresh gases, $Z_{F}$ reduces to $Z_{F}=\left(Y_{F}\right)_{u} /\left(v_{F} M_{F}\right)$, whereas $Z_{O}$ to $Z_{O}=\left(Y_{O_{2}}\right)_{u} / v_{O_{2}} M_{O_{2}}$. Therefore, $Z_{F} / Z_{O}$ is nothing but $\varphi_{u}$, the equivalence ratio of the fresh gases, since $Z_{F} / Z_{O}$ reduces in the unburnt gases of the flamelet to

$$
Z_{F} / Z_{O}=\left\{\left[Y_{F}\right]_{u} /\left[Y_{O_{2}}\right]_{u}\right\} \times\left\{v_{O_{2}} M_{O_{2}} / v_{F} M_{F}\right\}=\varphi_{u}
$$

Thus, as given by equation (10), the ratio $Z_{F}(x, y, t) / Z_{o}(x, y, t)$ allows us to derive an estimate of $\varphi_{u}$, the upstream mixture equivalence ratio, from any local composition within the flame (especially in the reaction zone). Heat release inside the flame thickness can then be adapted to $\varphi_{u}$ (within some uncertainties nevertheless since the assumption of equal diffusivities does not exactly hold). This approach will be used throughout the paper, which consequently considers the spray-flame as a set of local flamelets propagating in a heterogeneous medium. This concept requires that the characteristic length scale of composition variations is much larger than the flame thickness, which is the main assumption ruling the paper. Let us remark that in [30-31] is used another "invariant" which allows the authors to adapt heat release and activation energy in pace with the composition variation; the resulting one-step kinetics is implemented and checked with respect to a counter-flow diffusion flame.

From the quantitative point of view, it has been shown in [2] that the following general form of the adjustable quantity $\mathrm{F}\left(\varphi_{u}\right)$ gives reliable results for the single-phase premixture in what concerns the alkane/air flame speed and temperature 
where $\alpha$ is a coefficient depending on the fuel. The Zeldovich number has been determined theoretically for the studied fuel and more or less appeared as independent of the equivalence ratio. More precisely, for heavy alkane-air premixtures, it has been obtained $\alpha=0.33$ and $Z e=7$ [2, 29]. The quantitative comparison with experimental data (conducted in Ref. [2]) indicates that the model describes alkane spray combustion quite reasonably.

\section{Numerical experiments}

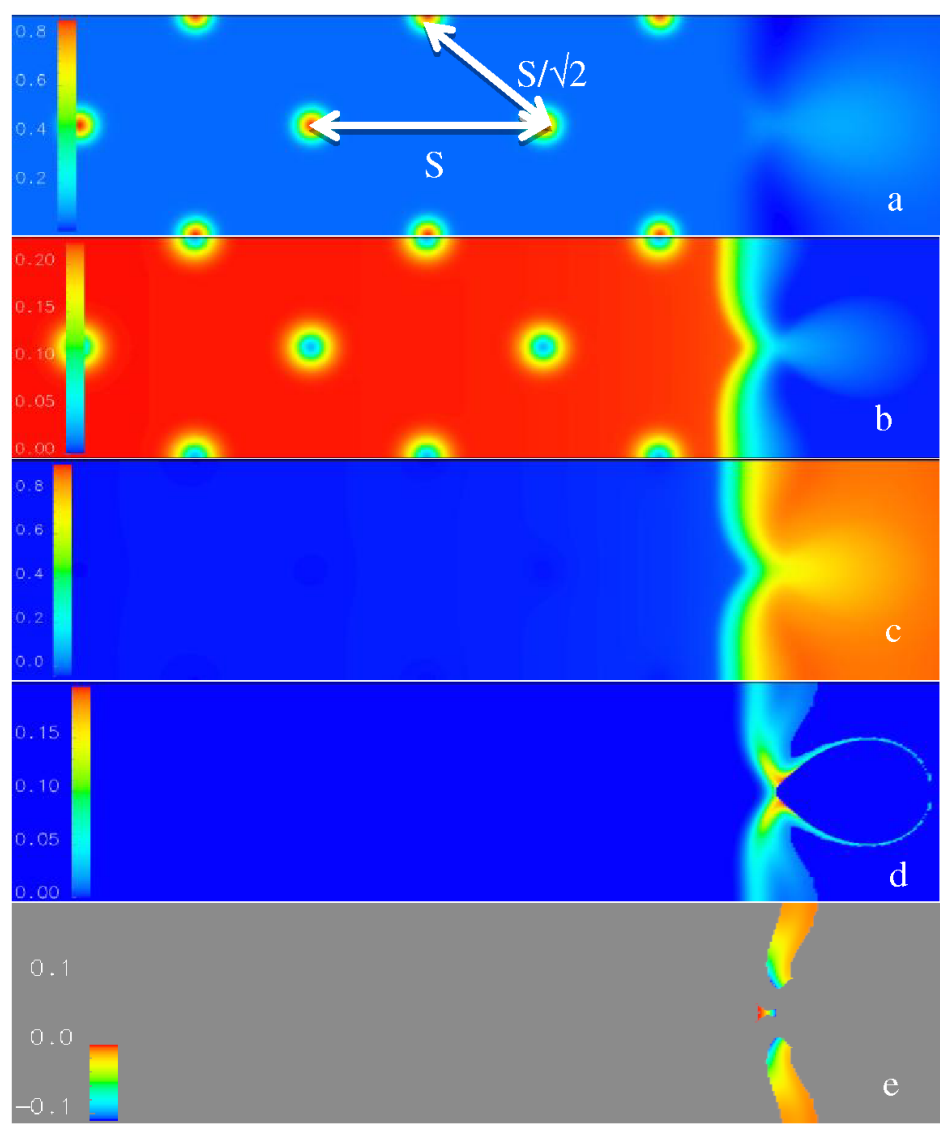

Figure 1: Simultaneous contour plots of fuel mass fraction (a), oxygen mass fraction (b), temperature (c), reaction rate $(\mathbf{d}), I_{W}^{-}$negative part of indexed reaction rate $(\mathbf{e})\left(s=6, \varphi_{G}=0.60, \varphi_{T}=1.25\right)$

The numerical experiments consist in solving the whole set of conservation laws, as presented in Appendix A. The numerical method [32] is briefly described in Appendix A. As mentioned before, the initial conditions correspond to a lattice of individual droplets surrounded by a mixture of fuel and air in equilibrium. Fig.1 presents the main characteristics of the numerical experiments. A spray-flame propagates from the right to the left. Note the snapshots correspond to the time when both triple flames meet and mark the 
end of a rapid stage in the spray-flame propagation process (the instant of the snapshots is indicated in Fig.2 by a vertical arrow). Spray-flame periodically consumes the droplets, which remain at rest until they start to vaporize as indicated in Fig.1.a, where the field of fuel mass fraction is represented. It also indicates that a certain amount of fuel is unburnt behind the (rich) spray-flame. Fig.1.b presents the field of oxygen mass fraction, while Fig.1.c corresponds to the temperature field. Fig.1.d shows the field of heat release. As for Fig.1.e, the negative part of the indexed reaction rate [33] is plotted for displaying the diffusion flames (see below); different elementary flames are noticeable, and will be discussed below.

The numerical experiments aim at determining the flame speed when combustion propagates through an array of fuel droplets positioned at the nodes of the face-centered lattice for a given rich spray. We are interested in the influence of the spray composition (i.e. $\varphi_{T}, \varphi_{G}$ ) and $S$, the lattice spacing. Droplet radius is obviously a function of liquid loading and lattice spacing. Three dimensionless lattice spacings are investigated: $s=3, s=6$ and $s=12$ (in units of stoichiometric premixed flame thickness, as described in Appendix A). Five different initial premixtures surrounding the droplets are considered: $\varphi_{G}=0, \varphi_{G}=0.2, \varphi_{G}=0.4$, $\varphi_{G}=0.6$ and $\varphi_{G}=0.8$. Evidently, when $\varphi_{G}$ is fixed, the amount of fuel initially under liquid phase increases as $\varphi_{T}$ increases. In the same manner, for fixed $S$ and $\varphi_{G}$, droplet radius increases as $\varphi_{T}$ increases.

For initiating combustion in the lattice, we follow the same procedure than that used in our previous works [1-2]: close to the open (right) end of the lattice, the temperature field is imposed with the profile of a singlephase flame. This allows us to vaporize and ignite the first droplet of the spray. To follow the combustion spread, we compute $\left\langle\theta>_{y}(x)\right.$, the mean temperature averaged in the periodic (y-)direction (i.e. transversally to the propagation). Then, we decide to define $x_{s f}$, the spray-flame position, as the locus where $<\theta>_{y}\left(x_{s f}\right)=0.5$.

\subsection{Spray-flame speed}

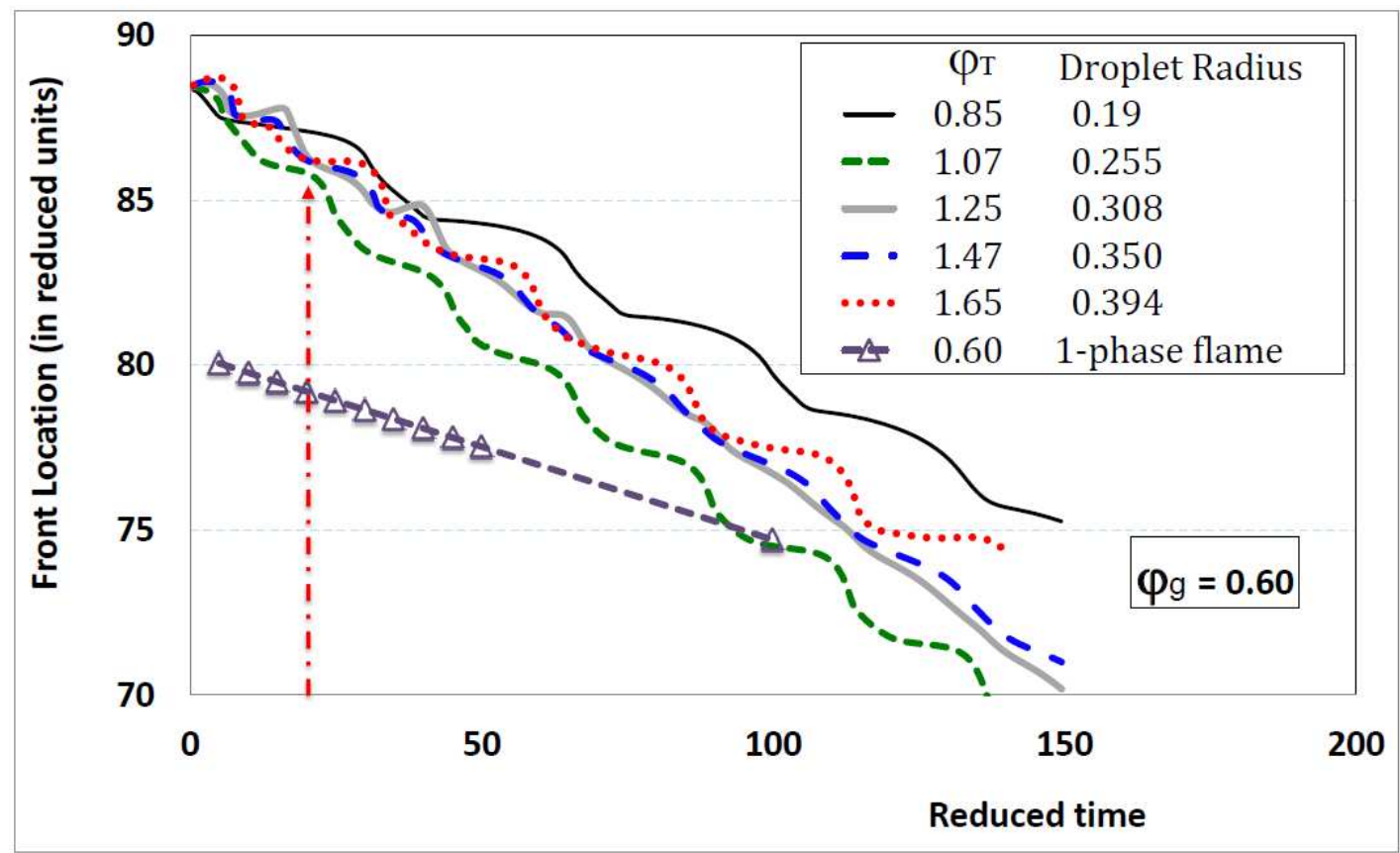


Figure 2: Front position vs. time for various liquid loading $\left(\varphi_{L}=0,0.25,0.47,0.65,0.87,1.05 ; \varphi_{G}=0.6\right.$; $s=6$ and iso-octane/air spray; droplet radius corresponding to each $\varphi_{L}$ is provided in units of stoichiometric premixed flame. Vertical arrow indicates the instant of the snapshots of Fig.1)

In this section, we present results on spray-flame speed at constant gaseous equivalence ratio $\varphi_{G}$. In other words, this numerical experiment considers a constant (saturated) vapour pressure; as the initial twophase mixture is supposed in equilibrium at a given temperature, the initial temperature is a logarithmic function of $\varphi_{G}$ thanks to the Clausius-Clapeyron relation of the considered fuel, as described in Appendix B. $\varphi_{T}$ is then modified by changing $\varphi_{L}$, the initial liquid loading. Considering the intermediate lattice spacing $s=6$, Figs. 2 and 3 show the flame front position for $\varphi_{G}=0.6$ and $\varphi_{G}=0.8$, respectively. Note that a single-phase premixed flame can propagate in both initial premixtures, slowly at $\varphi_{G}=0.6$ and more rapidly at $\varphi_{G}=0.8$.

In Figure 2, we can observe the actual way the flame propagates in the lattice for $\varphi_{G}=0.6$. The front position (defined as the transversally averaged isotherm $\left.<\theta>_{y}\left(x_{s f}\right)=0.5\right)$ is plotted versus time for various overall equivalence ratios (i.e. for increasing liquid loading). The lower (marked) curve corresponds to the front position of a single-phase premixed flame with the (initial) gaseous equivalence ratio (i.e. $\varphi_{L}=0, R=0$ ). The mean slope of each curve is here negative and its absolute value determines the flame speed for a given $\varphi_{T}$. It can be seen that, for this lattice spacing (s=6), the single-phase premixed flame with the overall (gaseous) equivalence ratio of 0.6 propagates significantly slower than all the considered sprayflames. One also observes that combustion propagates through the lattice in an unsteady manner [1], since it results from successive stages of droplet vaporization, species mixing, and reaction. Fig. 2 includes a lean case with the overall equivalence ratio $\varphi_{T}=0.85$ (i.e $\varphi_{L}=0.25$ ): this is the slowest spray-flame. It can be observed that the increase of the slope is not monotonous with the overall equivalence ratio. The maximum in slope is more or less obtained for the stoichiometric spray (i.e. $\varphi_{T}=1.07$ ). When liquid loading further increases the spray-flame speed decreases and seems to rapidly reach an asymptotic value. 


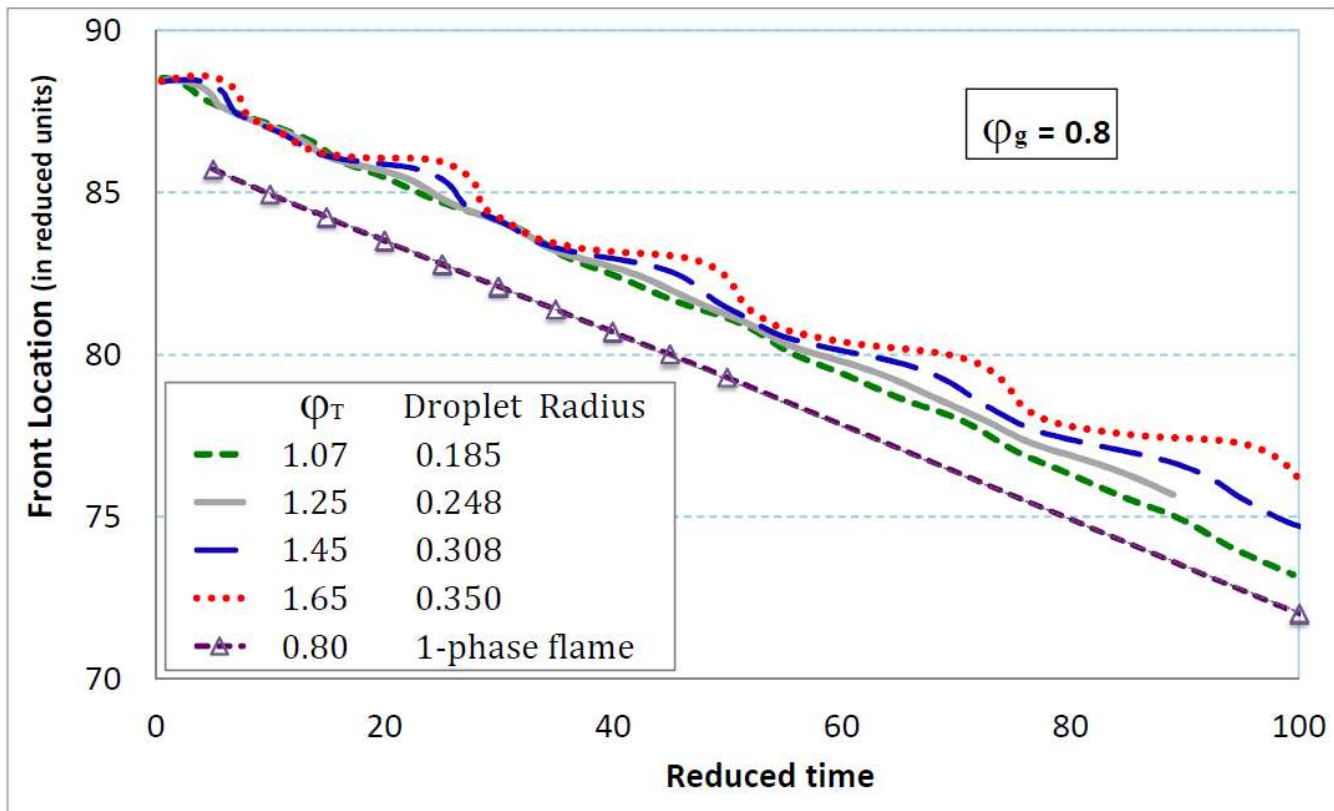

Figure 3: Front position versus time for various liquid loadings $\varphi_{L}=0,0.27,0.45,0.65,0.85$.

$$
\left(\varphi_{G}=0.8 \text { and lattice spacing } \mathrm{s}=6, \text { iso-octane/air spray }\right)
$$

Let us now consider Fig. 3, where the vapour pressure is chosen higher (i.e. $\varphi_{G}=0.8$ ). $U_{L}\left(\varphi_{G}=0.8\right.$ ), the speed of the single-phase flame that spreads in the initial saturated vapour is large. Then, unlike the previous case, the liquid loading is of little help in terms of propagation. The maximum spray-flame speed is again achieved for stoichiometric overall equivalence ratio. If the liquid loading is still increased the spray-flame speed slightly decreases. This slowdown with increasing equivalence ratio is, however, very weak in comparison with the single-phase premixed flame. This observation shows that fuel under liquid phase does not play the same role as the fuel under vapour. As we shall see, this role depends on the droplet size (i.e. mainly on the lattice spacing) and the initial vapour pressure. The fact that the droplet size plays a role in spray-flame propagation can already be seen for instance in Fig.3, where the fluctuations in flame front position increase as the liquid loading increases, i.e. as the droplet size increases. As studied in the previous contribution [1], those fluctuations in front position are due to the vaporization stage (if needed), which depends on the droplet radius squared.

We now investigate the role played by the saturated vapour (i.e. by $\varphi_{G}$ ) for the same intermediate lattice spacing $s=6$. In Fig. 4, the non-dimensional spray-flame speed is plotted with respect to $\varphi_{T}$, the overall equivalence ratio of the spray, for various equivalence ratios related to the vapour. In other words, the spray-flame speed is plotted for various (gaseous) equivalence ratio $\varphi_{G}$. In Fig.4, the curves of spray-flame speed are again compared with the single-phase premixed flame velocity. 


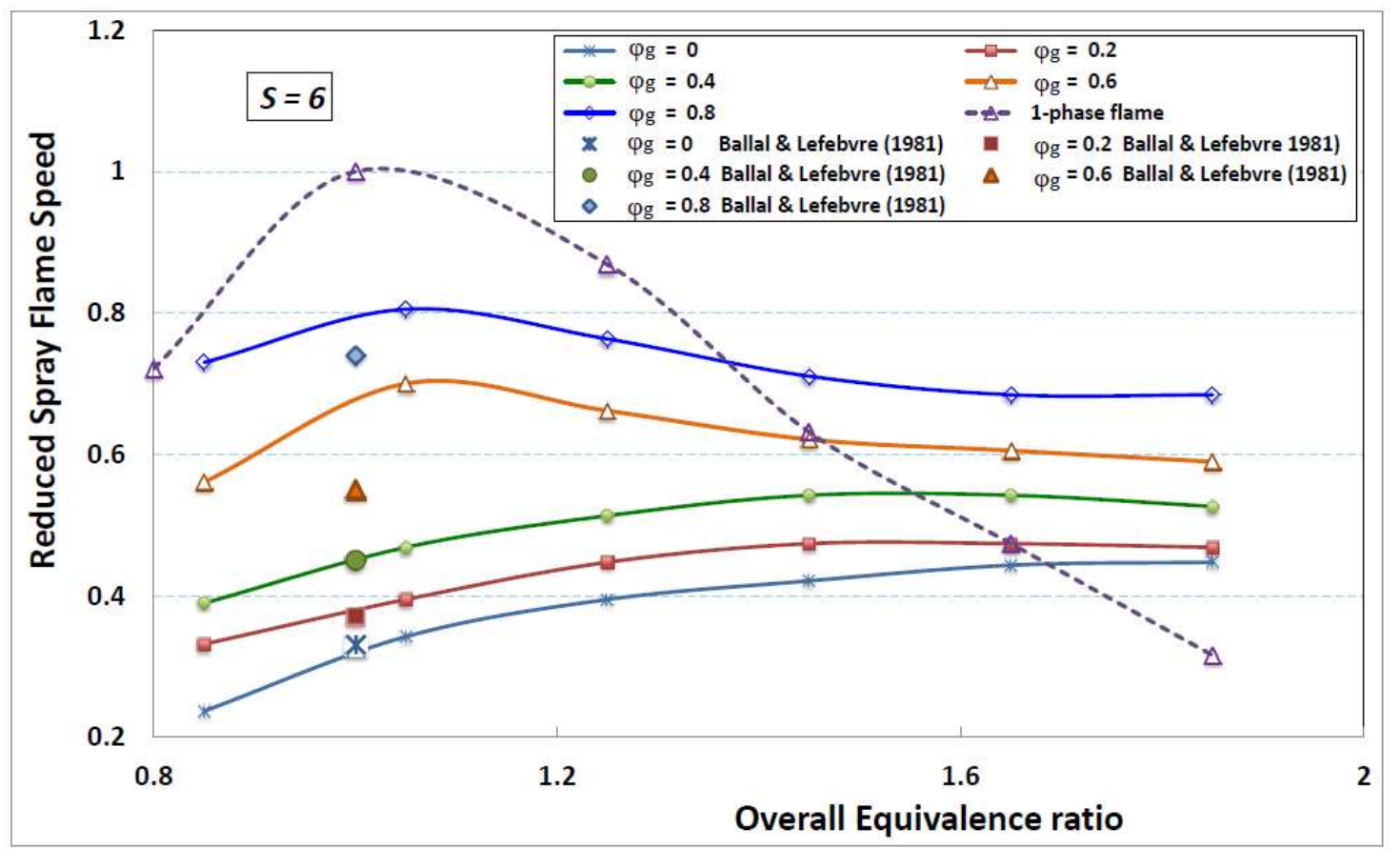

Figure 4: Spray-flame speed versus overall equivalence ratio of the spray

for different gaseous equivalence ratios ( $s=6$, heavy fuel oil/air spray;

numerical predictions are compared with the experiments by Ballal \& Lefebvre for $\varphi_{T}=1$ )

For the sake of validating the numerical approach, it can be checked that the experimental observations made in $[9,11,12]$ are retrieved by our numerical results:

- for moderately lean or stoichiometric sprays, the flame velocity is higher without droplets (the overall equivalence ratio being given). In Fig.4, we have added five experimental points from Ballal \& Lefebvre [9] for $\varphi_{T}=1$ (Sauter mean diameter in the experiment being in link with the lattice spacing used in our numerical predictions). The agreement between experiment and present modelling appears reasonably satisfactory.

- for rich sprays (say $\varphi_{T} \geq 1.6$ ), this situation reverses for all values of $\varphi_{G}$ : rich spray-flame speed is always higher than single-phase flame speed.

It is worth noting that for very low vapour pressure (i.e. $\varphi_{G}=0$ ) a spray-flame does exist, even though its velocity is quite low. In other words, combustion ensures vaporization at a pace that allows its own propagation! From Fig.4 (i.e. for $s=6$ ), we additionally have to underline another trend: for $\varphi_{G}=0,0.2,0.4$, i.e. when $\varphi_{G}$ corresponds to a non-flammable single-phase mixture (in other words, combustion needs droplets to propagate), the spray-flame speed is an increasing function of $\varphi_{T}$. Otherwise, for higher $\varphi_{G}$, the spray-flame speed exhibits a maximum in the vicinity of the (overall) stoichiometry. Lastly, we observe that, when the overall equivalence ratio still increases, the spray-flame velocity does not 
vary so much. In other words, on the far rich side, it seems that a constant amount of liquid fuel is involved in spray-flame propagation.

\subsection{Role of the lattice spacing}

In the previous paragraphs, increasing the liquid loading led us to slightly increase the droplet radius (in 2D, as the square root of $\varphi_{L}$ ). A more efficient increase in droplet radius is achieved when the lattice spacing is enhanced: for fixed $\varphi_{L}$, the droplet radius increases linearly with $S$. For three different values of gaseous equivalence ratio $\left(\varphi_{G}=0.2 ; 0.6 ; 0.8\right)$, the role played by the lattice spacing is now studied. More precisely, for each $\varphi_{G}$, the spray-flame velocity is estimated as a function of the overall equivalence ratio for three lattice spacings (s=3, 6 and 12). Figure 5 (resp. 6 and 7) plots the spray-flame speeds for $\varphi_{G}=0.2$ (resp. for $\varphi_{G}=0.6$ and $\varphi_{G}=0.8$ ). On each figure, the dashed line recalls the 1-phase flame speed versus gaseous equivalence ratio (since $\varphi_{G}=\varphi_{T}$ ), while the experimental data from Ballal \& Lefebvre [9] are reported for $\varphi_{T}=1$ [Note the Sauter mean diameter studied in the experiment corresponds to $s=10$ and not to $s=12]$.

The spray-flames characterized by $\varphi_{G}=0.2$ need to vaporize droplets, previously to any propagation. Hence, Fig. 5 indicates an important role played by lattice spacing on the spray-flame speed. For small lattice spacing (say, $s=3$ ), droplet radius being small, the droplets quickly vaporize and contribute to achieve a rather rapid propagation. For larger lattice spacings $(s=6 ; s=12)$, droplet radius being higher, the vaporization is slower and imposes its pace to the combustion. When the overall equivalence ratio is high, Fig. 5 indicates that propagation velocity of the spray-flame with small lattice spacing [i.e. $s=3$ ] follows the same trend as the single-phase flame: spray-flame speed decreases as equivalence ratio increases. This feature is due to the rapid vaporization and mixing of the fuel, which then burns accordingly with the chemical scheme. 


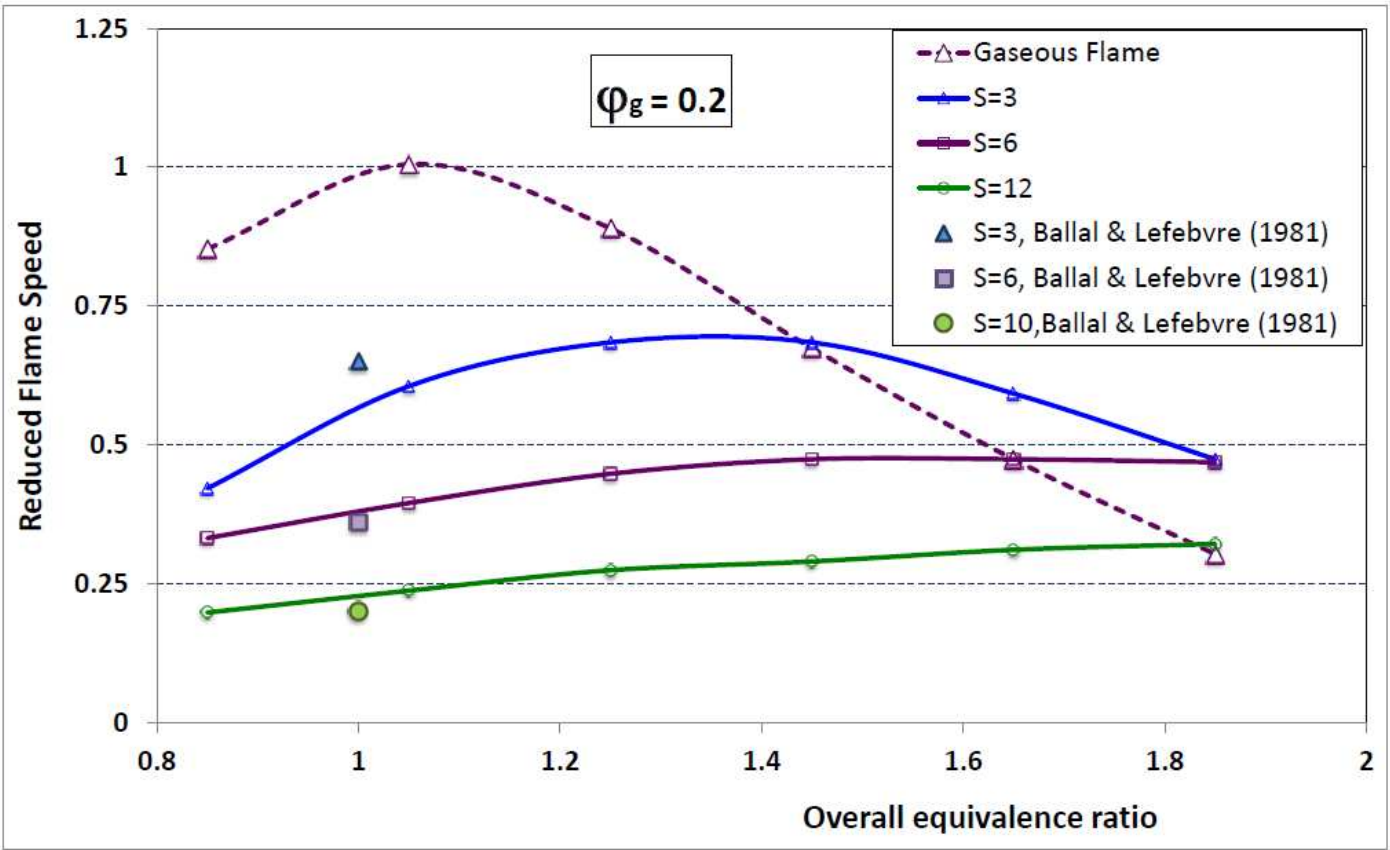

Figure 5: Lattice spacing effect on spray-flame velocity:

spray-flame speed versus overall equivalence ratio for $\mathrm{s}=3,6$ and 12 and $\varphi_{G}=0.2$

(comparison with the experiments by Ballal \& Lefebvre for $\varphi_{T}=1$, heavy fuel oil/air spray)

Figure 6 treats of a slightly different situation since vapour pressure is high enough $\left(\varphi_{G}=0.6\right)$ to obtain a flammable (initial) single-phase premixture. The spray-flame now requires a smaller amount of fuel coming from the liquid loading to reach the near-stoichiometric conditions in the premixture. Therefore, for relatively small droplet size (cases $s=3$ or $s=6$ ), the spray-flame presents a maximum of its velocity. The intensity of the maximum is nevertheless lower than the maximum single-phase flame speed, since non-zero vaporization time scale impacts on combustion. The latter impact is weaker for small lattice spacing: for $s=3$, the curve of spray-flame speed roughly adopts the same pattern than that of the single-phase flame.

On the very rich side of the overall equivalence ratio, spray-flame with small lattice spacing again experiences the rich chemistry, whereas the spray-flame with large lattice spacing, i.e. with a large radius, maintains a large part of the liquid fuel non reacting. In other words, although vaporization of large droplets is a slow process, the rich spray-flame with large droplets propagates faster than the rich spray-flame with small droplets.

Figure 7 presents the spray-flame speed when the vapour pressure is large (i.e. $\varphi_{G}=0.8$ ). If the lattice spacing (or the droplet size) is small, the liquid loading rapidly participates to the spray-flame propagation. In this case (say, $s=3$ ), the spray-flame shares the same trends as the single-phase flame: the maximum flame speed occurs at (overall) stoichiometry, and a flammability limit occurs on the rich side, too. Otherwise, for large droplets (say, $s=12$ ), the spray-flame propagation ignores the liquid loading and propagates at the same speed as would propagate a single-phase flame in the saturated vapour [since $U_{L}\left(\varphi_{G}=0.8\right)=0.76$, as discussed below]. 
The previous comments on Figs. 5-7 invite us to stress on the following features, that have already been observed in numerous experimental and numerical studies [11, 12, 20, 21, 23, 34] :

- $\quad$ spray with small droplets (i.e. short vaporization time scale) tends to burn with the same trends as the single-phase premixture.

- when the overall equivalence ratio is large enough (say, $\varphi_{T} \geq 1.5$ ) spray-flame speed is always higher than the single-phase flame which tends to extinguish according to the single-step chemical scheme. The difference between spray-flame and single-phase flame becomes more pronounced as droplet size increases. In other words, the presence of large droplets causes some amount of fuel (i.e. under liquid phase) to be maintained out of the combustion. This point will be clarified below.

Furthermore, the vaporization time scale of the droplets undoubtedly plays a role in the spray-flame propagation. Since droplet radius depends on both lattice spacing and liquid loading, the point deserves to be studied specifically. This is the object of the next paragraph. 


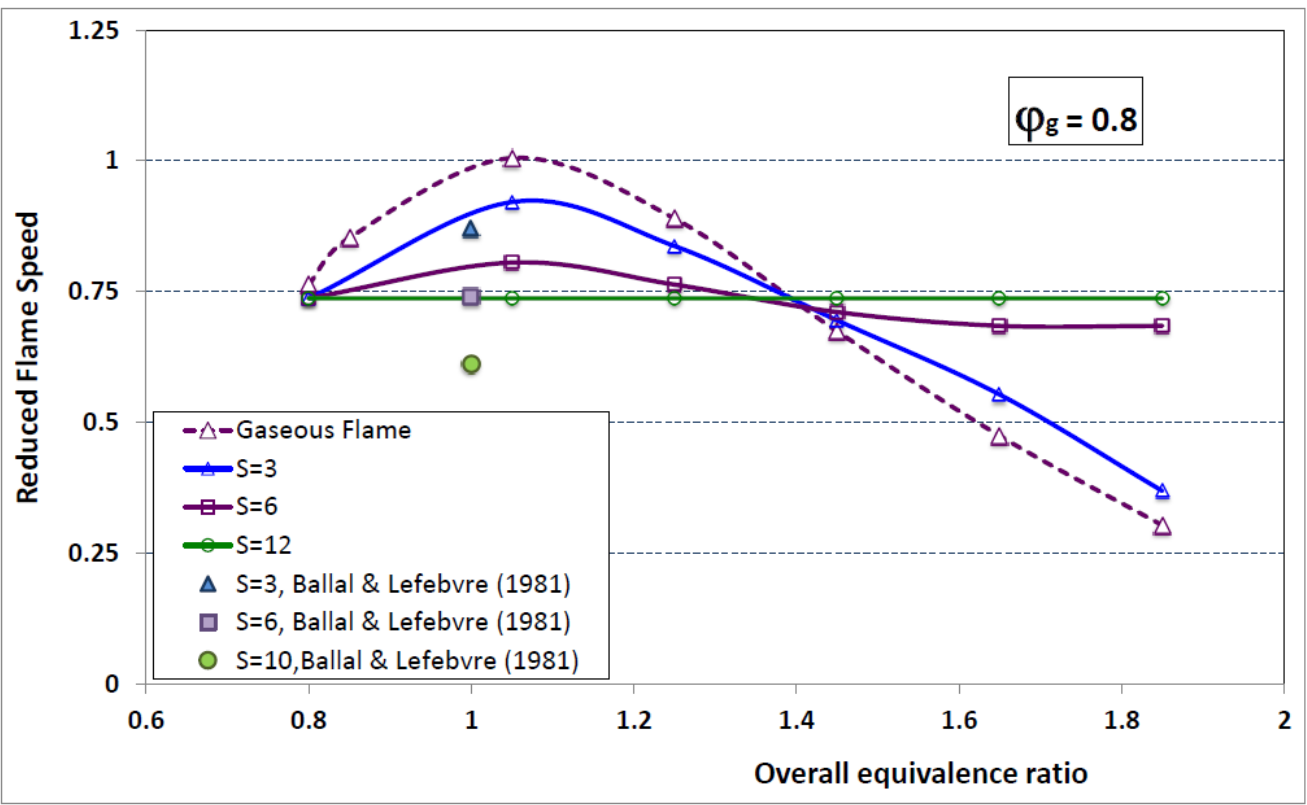

Figure 7: Lattice spacing effect on the spray flame velocity:

velocity versus overall equivalence ratio for $s=3,6$ and 12 and $\varphi_{G}=0.8$

(comparison with the experiments by Ballal \& Lefebvre for $\varphi_{T}=1$, heavy fuel oil/air spray)

\subsection{Detailed role of droplet radius}

We now consider a spray with fixed temperature and given chemical composition: $\varphi_{T}$, its overall equivalence ratio, is hence determined, as well as $\varphi_{G}$, the equivalence ratio related to its saturated vapour. Different lattice spacings are considered. We present a series of figures (i.e. Figs. 8-10) for sprays with a fixed overall equivalence ratio. In each figure, a certain amount of fuel is supposed in vapour form and the rest under liquid state; the size of the corresponding fuel droplet is a parameter still free. Our numerical study then consists in computing the spray-flame speed dependence on the radius of these fuel droplets.

Figure 8 concerns a slightly rich spray $\left(\varphi_{T}=1.25\right)$. It presents the spray-flame speed versus droplet radius for various gaseous equivalence ratios (or various vapour pressures). For this case of overall equivalence ratio, the results show that the single-phase flame speed (i.e. $U_{L}\left(\varphi_{T}=1.25\right)=0.89$ ) is always larger than any twophase flame of the same overall composition. Let us observe additionally that three curves (i.e. for $\varphi_{G}=0 ., 0.2,0.4$ ) correspond to a non-flammable gaseous surrounding of the droplets. It is noticeable that these curves are close to each other. In other words, the spray-flame needs to resort to vaporization of some liquid fuel. In that case, vaporization rules propagation, and the spray-flame speed behaves in inverse ratio to droplet radius (as already studied in lean spray, see Ref. [1]).

Furthermore, let us recall that the initial vapour (in fig.8) is only flammable for $\varphi_{G}=0.6$ and $\varphi_{G}=0.8$, for which the corresponding single-phase flame speeds are $U_{L}\left(\varphi_{G}=0.6\right)=0.29$ and $U_{L}\left(\varphi_{G}=0.8\right)=0.76$, 
respectively. Therefore, from Fig.8, it is clear that droplet vaporization enhances flame speed, whatever the droplet size and the considered vapour pressure. But, this enhancement reaches the single-phase flame speed of the same overall composition, only for vanishing droplet size. To sum up, Fig. 8 is characteristic of the situation for $U_{L}\left(\varphi_{T}\right)>U_{L}\left(\varphi_{G}\right)$, where $U_{L}\left(\varphi_{T}\right)$ represents the laminar flame speed of the single-phase premixture obtained for $\varphi=\varphi_{T}$ (which is also the spray-flame speed for a spray with infinitely small droplets)

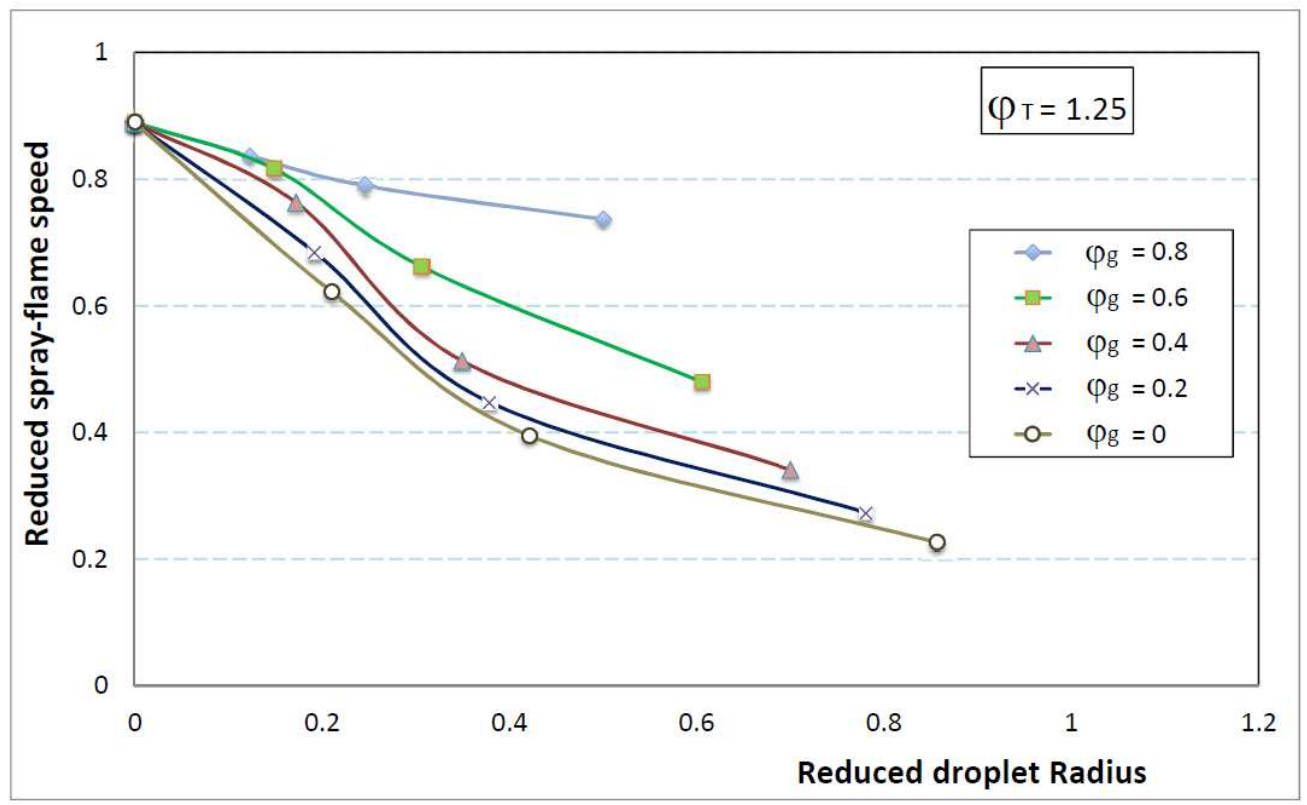

Figure 8: Spray-flame speed versus droplet radius for various $\varphi_{G}\left(\right.$ and $\left.\varphi_{T}=1.25\right)$.

Figure 9 now concerns a moderately rich spray $\left(\varphi_{T}=1.45\right)$. Spray-flame speed is again plotted versus droplet radius for various vapour pressures in this figure, which exhibits two different behaviours. The first case corresponds to the results obtained for $\varphi_{G}=0.8$, for which we have $U_{L}\left(\varphi_{G}=0.8\right)=0.76$ and $U_{L}\left(\varphi_{T}=1.45\right)=0.67$. This situation [i.e. for $\left.U_{L}\left(\varphi_{G}\right)>U_{L}\left(\varphi_{T}\right)\right]$ exhibits a flame speed enhancement with droplet radius. This behaviour is explained thanks to the following rationale: for very small droplets $(R \rightarrow 0)$, vaporization and mixing are immediate so that we start from the rich single-phase case with the speed $U_{L}\left(\varphi_{T}=1.45\right)=0.67$. On the other hand, for large droplets we retrieve the single-phase with the speed $U_{L}\left(\varphi_{G}=0.8\right)=0.76$. In other words, the droplets are large enough to have no time to fully vaporize during the spray-flame propagation. This is the sense of the upper curve of Fig. 9 (i.e. for $\varphi_{G}=0.8$ ), where spray-flame speed increases with droplet radius: the larger the droplets, the lesser liquid fuel is involved in combustion. 


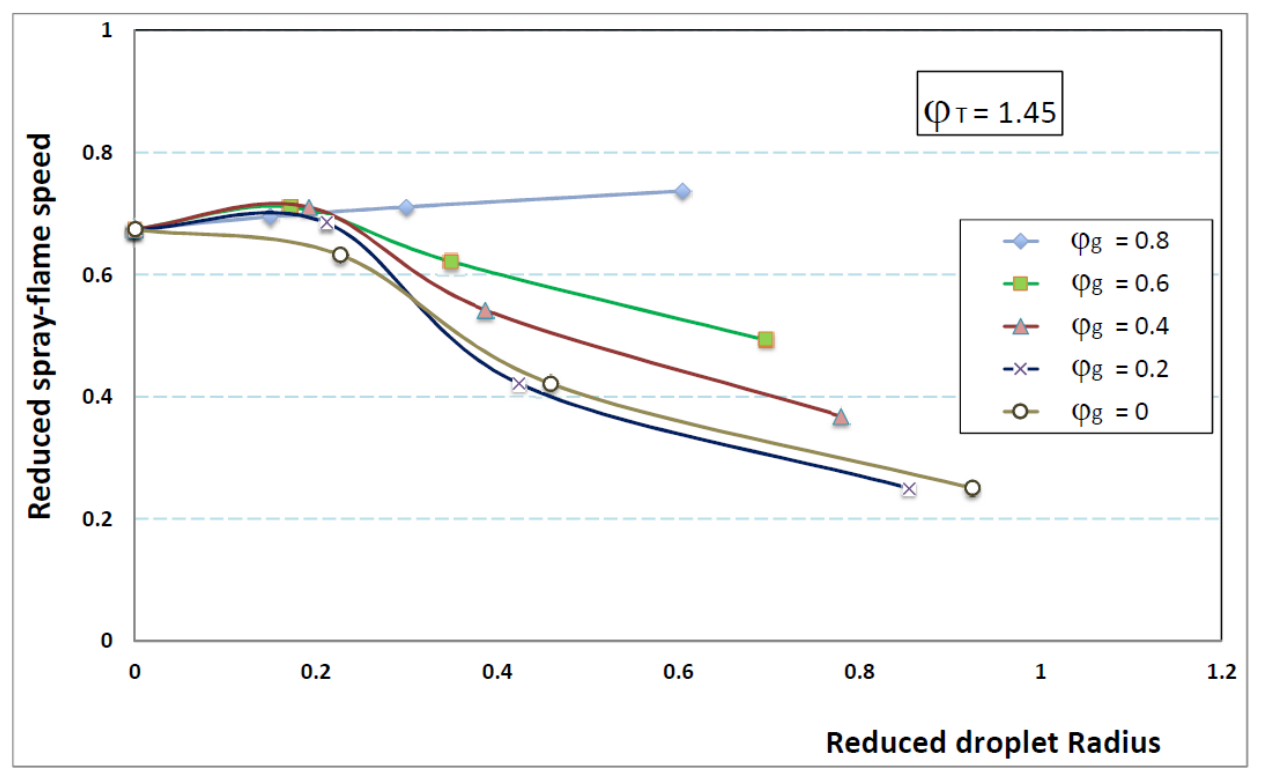

Figure 9: Spray-flame speed versus droplet radius for various $\varphi_{G}\left(\right.$ and $\left.\varphi_{T}=1.45\right)$.

In fig.9, the second case considers the other four curves $\left(\varphi_{G}=0 ., 0.2,0.4,0.6\right)$, for which $U_{L}\left(\varphi_{G}\right)<U_{L}\left(\varphi_{T}\right)$. These curves are non-monotonic as droplet size increases: if droplet radius slightly increases, less liquid fuel is involved in combustion, and the spray-flame speed slightly increases; now, if the droplet radius is too large, the spray-flame speed asymptotically tends to $U_{L}\left(\varphi_{G}\right)$, since the vaporization time tends to infinity; therefore, each curve reaches a maximum for an intermediate droplet radius, and afterwards decreases to the asymptotic flame speed $U_{L}\left(\varphi_{G}\right)$ [which can be a zero value].

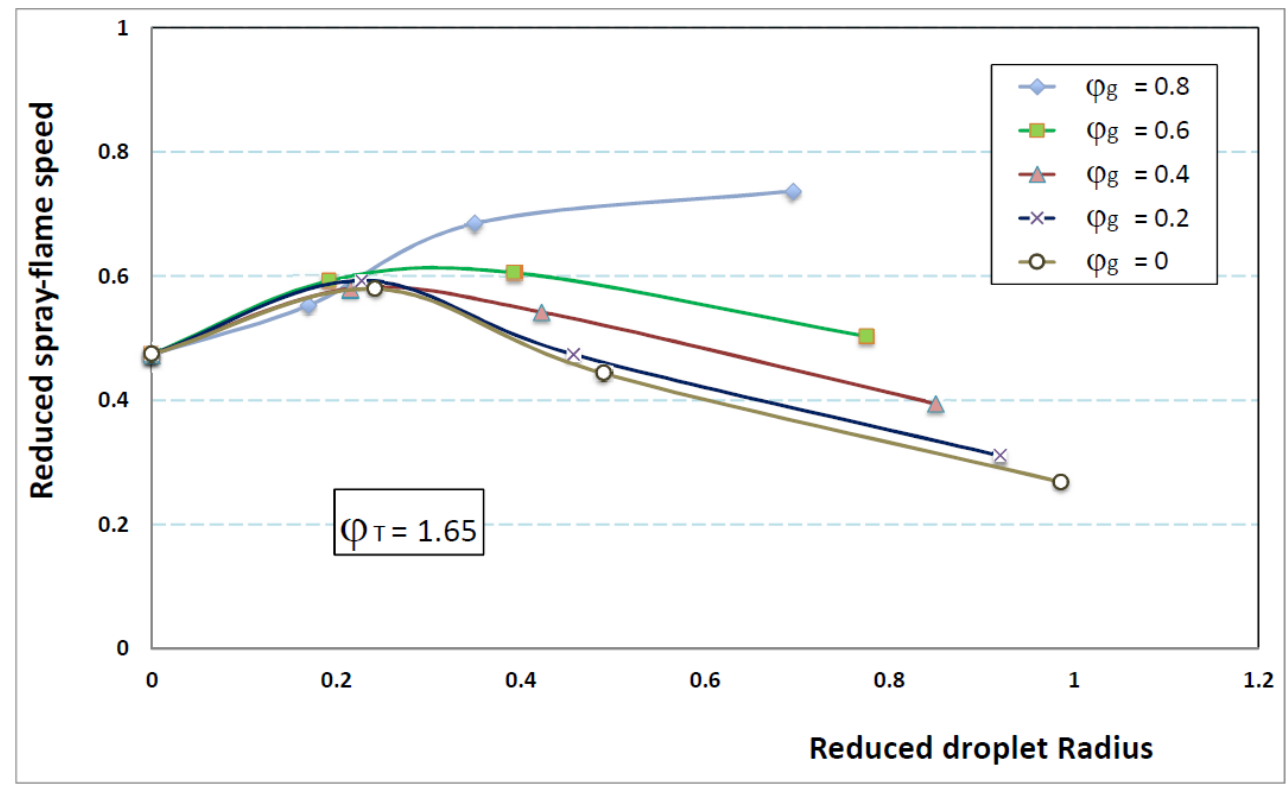

Figure 10: Spray-flame speed versus droplet radius for various $\varphi_{G}$ (and $\varphi_{T}=1.65$ ). 
It is interesting to still increase the overall equivalence ratio of the spray. Fig.10 is concerned with a rich spray $\left(\varphi_{T}=1.65\right)$ : it plots spray-flame speed versus droplet radius for various vapour pressures. In a more marked manner, this case presents the same trends as those of Fig. 9. Initial configurations with feeble vapour pressure need droplet vaporisation: if the droplets are vanishing, a very rich premixture burns; by contrast, if the droplets are very large, only a lean vapour pressure is submitted to combustion. Both opposite trends explain why spray-flame speed versus droplet radius here exhibits a maximum for an intermediate droplet radius. On the other hand, if the initial configuration has an equivalence ratio of vapour background which does not require droplet vaporisation for efficient burning, the presence of large droplets allows the actual mixture to ignore the liquid loading; this is why the curve with $\varphi_{G}=0.8$ is an increasing function of droplet radius, this curve starting from $U_{L}\left(\varphi_{T}=1.65\right)=0.48$ (for vanishing droplets) and ending at $U_{L}\left(\varphi_{G}=0.8\right)=0.76$ (for large droplets). This confirms that liquid loading does not intervene when the droplets are huge.

To sum up, for fixed $\varphi_{T}$ and $\varphi_{G}$ (with $\varphi_{T}>1>\varphi_{G}$ in the present work), increasing the droplet radius can have different effects :

- if $U_{L}\left(\varphi_{T}\right)<U_{L}\left(\varphi_{G}\right)$

and $U_{L}\left(\varphi_{G}\right) \square U_{L}^{*}$, spray-flame speed is enhanced (and tends to $U_{L}\left(\varphi_{G}\right)$ as $P e_{s} \square 1$ )

and $U_{L}\left(\varphi_{G}\right) \square U_{L}^{*}$, spray-flame speed admits a maximum for an optimal droplet radius.

- if $U_{L}\left(\varphi_{T}\right)>U_{L}\left(\varphi_{G}\right)$

and $U_{L}\left(\varphi_{T}\right) \square U_{L}^{*}$, spray-flame speed diminishes (and tends to $U_{L}\left(\varphi_{G}\right)$ as $P e_{s} \square 1$ )

and $U_{L}\left(\varphi_{T}\right) \square U_{L}^{*}$, spray-flame speed admits a maximum for an optimal droplet radius.

We now turn to a detailed description of how the flame propagates. 


\section{Analysis of spray-flame structure}

\subsection{Large lattice spacing}

In the case of lean sprays, it has been found [1] that the flame propagated from one droplet to the next in different ways, either with a very lean premixed flame, or with constant re-ignition of the next droplet in the case of very small equivalence ratio relative to initial vapour. Once the next droplet was ignited in one way or another, a triple flame propagated around the droplets, leaving behind the diffusion flame around the droplet. Such a propagation was found to correspond to the third propagation mode observed in [19].

In the rich case, however, Roland Borghi suggested -see the sketches in the book by Borghi and Champion [35] and also in Demoulin and Borghi [36] - that the diffusion flame should be around the oxidant; this was called pocket combustion. Let us see what we obtain in the present simulations.

In Fig. 11, we show different fields characteristic of the spray-flame at a particular instant for a the large lattice spacing $s=12$ : fuel and oxygen mass fractions, temperature, heat release. The fifth field requires a particular comment: we have plotted $I_{W}^{-}$, the negative part of the indexed reaction rate, which is defined as follows [33]

$$
\left\{\begin{array}{ll}
I_{W}^{-}=0 & \text { if } I_{T} \geq 0 \\
I_{W}^{-}=W \times I_{T} /\left|I_{T}\right| & \text { if } \quad I_{T}^{<0}
\end{array} \quad \text { with } I_{T}=\bar{\nabla} \psi_{F} \cdot \bar{\nabla} \psi_{O}\right.
$$

where $I_{T}$ is the so-called Takeno flame index [37]. $I_{T}$ is expected negative when the species diffuse towards each other, as it occurs in a diffusion flame. In other words, plotting the negative part of $I_{W}$ helps us to localize the diffusion flames. The chosen time in Fig. 11 corresponds to the late instants of the triple flame stage (i.e. just before both triple flames meet); we do not actually show the complete computational domain. As in the lean case [1], for a large value of $s$, we observe triple flames, the wings of which are connected here to lean and rich premixed flames. Fig. 11.e clearly exhibits the location of the diffusion flames. The first diffusion flame accompanies the triple flame, while the second one burns oxygen in excess between the previous vaporized droplets. As a result, the lean premixed flame does not burn all the oxidant available locally, and is followed by a diffusion flame surrounding the oxidant (and not the fuel as in the lean case), as suggested by Borghi. We are here at a late stage of droplet vaporization, it appears that a significant premixture exists close to the droplet (we recall that the diffusion coefficient is larger for the oxidant than for the fuel, i.e. a Lewis number of 0.9 for the oxidant and 1.8 for the fuel). As a matter of fact, the flame around the droplet is a (slow) rich premixed flame. This rich premixed flame does not burn all the fuel, since a significant amount of fuel is found at the right of the figure where the flame extinguishes. In the same manner, the premixed flame does not burn all oxygen: in Fig. 11.c, a feeble amount of oxygen is present at the right of the domain, and is slowly consumed thanks to the diffusion flame located at the right of the domain. 


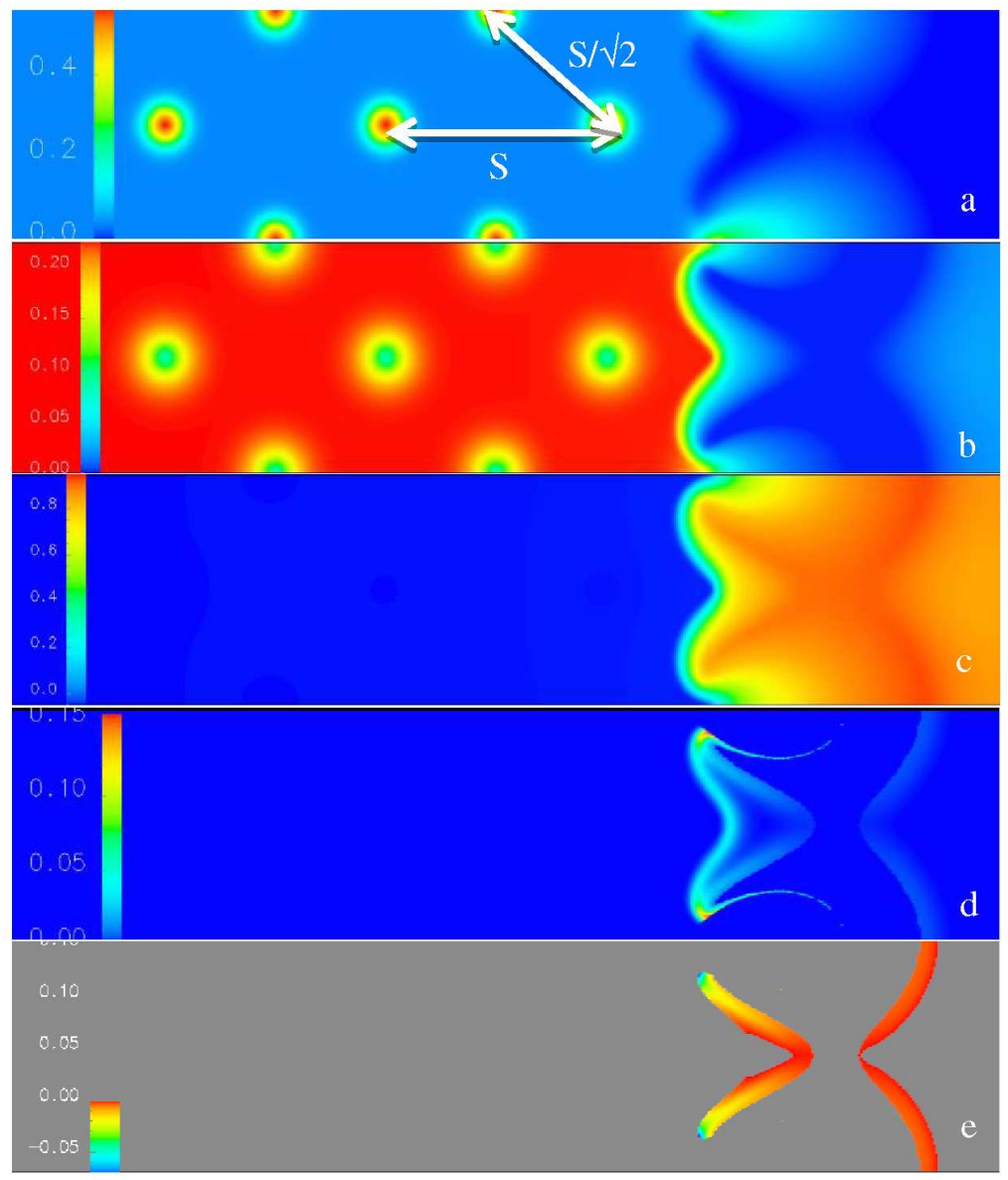

Figure 11: Simultaneous contour plots of fuel mass fraction (a), oxygen mass fraction (b), temperature (c), reaction rate $(\mathbf{d}), I_{W}^{-}$negative part of indexed reaction rate $(\mathbf{e})\left(s=12, \varphi_{G}=0.60, \varphi_{T}=1.25\right)$

As in the case $s=6$ (the case presented in Fig.1), the case $s=12$ with a value of the vapour equivalence ratio low enough, the flame propagates from one droplet to the other by re-ignition of the next droplet, which Cekalin [4] called time relay combustion (see also the mode 3 in Ref.19). Note that this mechanism leads to a faster propagation velocity than the premixed flame for very rich $\varphi_{T}\left(\right.$ say $\left.\varphi_{T}>1.65\right)$. 


\subsection{Small lattice spacing}

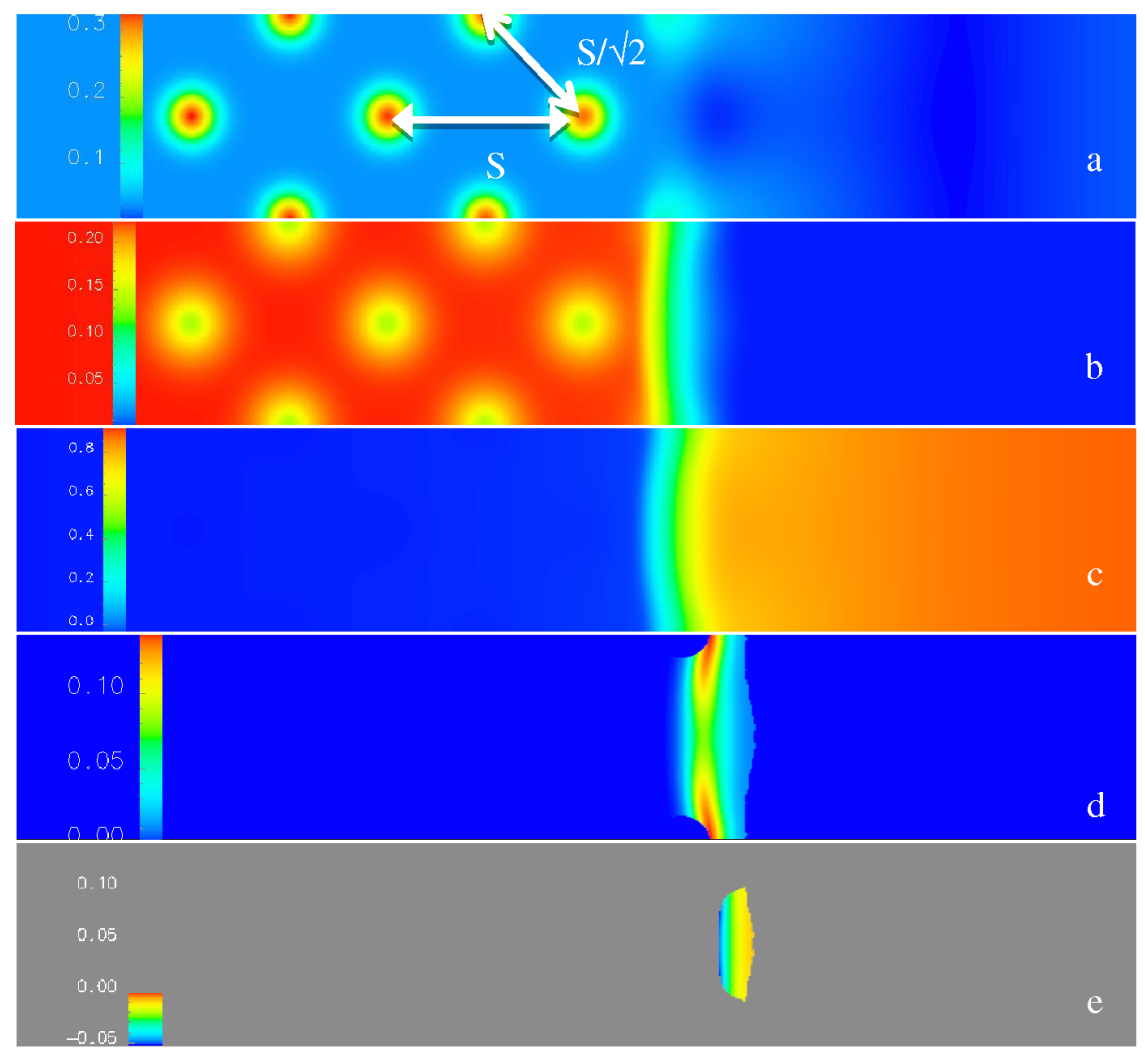

Figure 12: Simultaneous contour plots of fuel mass fraction (a), oxygen mass fraction (b), temperature (c), reaction rate $(\mathbf{d}), I_{W}^{-}$, the negative part of indexed reaction rate $(\mathbf{e})\left(s=3, \varphi_{G}=0.60, \varphi_{T}=1.25\right)$

In principle, for a very small lattice spacing [i.e. $s \approx 0$ ], the flame propagation is supposed to retrieve the homogenization regime as studied in [22] and [26-27]. Fig.12 presents the same plots as in Fig.11, fuel and oxidant mass fractions, temperature, heat release, and the negative part of indexed reaction rate, for the smallest value of $s$ that we have studied (i.e. $s=3$ in units of stoichiometric single-phase flame thickness). In Fig.12, we observe that combustion does not yet occur in a completely pre-vaporized case. If one only looks at both temperature and oxidant fields, it might be concluded that the flame appearance is that of a premixed flame. By contrast, heat release field is rather strange, with a reaction zone thickness very large, of the order of the flame thickness.

To better understand what happens, let us look at the fuel mass fraction. At the time step chosen here, we are in the late stage of burning of one droplet, and the reaction proceeds around the droplet, reminiscent of the triple flame in Fig.11. It seems actually (see the blue zone with almost no fuel mass fraction) that the diffusion flame exists but is not separated from the rest of the reaction. This is obviously corroborated by Fig. 12.e. The overall reaction zone thickness here appears very large: it corresponds to both premixed and 
diffusion flames. As a result, for $s=3$ there is no more complete separation between both types of flames, an effect we have already observed in the lean case (see Fig. 8 of Ref. [1]).

\section{Conclusion}

In the paper, which follows a previous article on lean sprays, we numerically study the propagation of spray-flames in a rich spray, which is initially schematized by a regular lattice of droplets. The paper focus is the spray-flames characterized with a rich overall equivalence ratio (i.e. $\varphi_{T} \geq 1$ ). The characteristics of the spray-flames are expressed in units of the stoichiometric single-phase flame.

We aim at comparing our results with the characteristics of the classical single-phase premixed flame that has the same overall equivalence ratio, the speed of which has been denoted by $U_{L}\left(\varphi_{T}\right)$. This corresponds to the "all fuel flame" that propagates (if any) in the mixture obtained when all fuel is vaporized and mixed. Another single-phase premixed flame of reference is the flame which would exist if the saturated (initial) vapour burns only. The latter single-phase flame is called "initial vapour flame", and is characterized by $\varphi_{G}$, the so-called gaseous equivalence ratio, and its velocity has been denoted by $U_{L}\left(\varphi_{G}\right)$. It has been argued in the literature (see Ref. [7]) that the relevant propagation velocity in spray is precisely the singlephase flame speed $U_{L}\left(\varphi_{G}\right)$. The whole set of our results do not corroborate this claim: it presents rather intricate data that strongly depend on the three parameters $\varphi_{G}, \varphi_{T}$, and $s$, the lattice spacing. For the studied situations (i.e. $0 \leq \varphi_{G}<1<\varphi_{T} \leq 1.85$ ), we can conduct the following discussion.

Here, the simplest case to interpret is the case $P e_{s} \gg 1$ (i.e. $\tau_{\text {prop }} \square \tau_{\text {vap }}$ ): droplet vaporization does not act on propagation. In other words, the liquid loading is maintained apart from the combustion that rules propagation (a part of the liquid fuel possibly reacting with the remaining oxygen behind the leading front). We here retrieve the situation described by Hayashi et al. [7], who claimed that the relevant propagation velocity is $U_{L}\left(\varphi_{G}\right)$, i.e. the propagation due to the saturated vapour, only. In our present study, this is the situation met for $\varphi_{G}=0.8$ and large droplet radius.

By contrast, if $P e_{s} \leq O(1)$ (i.e. $\tau_{\text {prop }} \geq \tau_{\text {vap }}$ ), propagation uses (or must use) some part of the liquid loading. Thus, propagation results from the following mechanisms: vaporization is needed to increase a too lean $\varphi_{G}$, which diminishes $\tau_{\text {prop }}$ (since $U_{L}$ increases); as soon as $\tau_{\text {prop }}<\tau_{\text {vap }}$, vaporization ceases to contribute to propagation. Two subcases are then to be considered here: (a) if $U_{L}\left(\varphi_{T}\right)$ is of the same order as $U_{L}^{*}$ [i.e. $U_{L}\left(\varphi_{T}\right) \approx O(1)$ in our figures], spray-flame speed is a decreasing function of droplet radius, and asymptotically reaches $U_{L}\left(\varphi_{G}\right)$ for large droplets ; (b) if $U_{L}\left(\varphi_{T}\right)$ is small or moderate, there is an optimal droplet radius for propagation, and, for radii larger than the optimal radius, the spray-flame speed presents a minor dependence on $\varphi_{T}$. Subcase (b) includes the most striking situation: we have found propagation even for $U_{L}\left(\varphi_{G}=0\right)=U_{L}\left(\varphi_{T}=2.2\right)=0$ [i.e. neither "initial vapour flame", nor "all fuel fame" exist], the 
optimal radius being on the order of 0.4 times the stoichiometric flame thickness, and the maximum sprayflame speed being found close to 0.45 .

As for the comparison with the experimental literature, it must be conducted in terms of the fundamental laminar spray-flame speed, because our DNS carried out at the scale of the droplets is not able to simulate large scale instabilities, or spherical expansion instabilities as those occurring in the Wilson chambers. As mentioned in Introduction, those instabilities are very sensitive to perturbations: a change in geometry, pressure, can trigger those instabilities, and increase the measured propagation velocity. This is a possible reason for the conflicting results found in the experimental literature. Whatever the scattering in literature, our results corroborate an often observed feature on spray-flames: the rich flammability of sprayflames can be enhanced by a large extent, provided that droplet radius belongs to the appropriate range.

\section{Acknowledgements}

The present work has received the support of the Research Program "Micropesanteur Fondamentale et Appliquée" GDR n²799 CNRS/CNES under the contract CNES/140569.

\section{Appendix A}

This addendum is devoted to derive a non-dimensional form of the governing equations from the basis conservation laws, which read for energy and species

$$
\begin{gathered}
\frac{\partial\left(\rho C_{p} T\right)}{\partial t}+\operatorname{div}\left(\bar{V} \rho C_{p} T\right)=\operatorname{div}(\lambda \bar{\nabla} T)+Q w\left(\rho, Y_{i}, T\right) \\
\frac{\partial Y_{i}}{\partial t}+\operatorname{div}\left(\bar{V} \rho Y_{i}\right)=\operatorname{div}\left(\rho D_{i} \bar{\nabla} Y_{i}\right)-v_{i} M_{i} w\left(\rho, Y_{i}, T\right) \\
\text { where } \quad w\left(\rho, Y_{i}, T\right)=(\rho)^{2} Y_{F} Y_{O} \exp \left[-T_{A} / T\right]
\end{gathered}
$$

is the reaction rate, and $T_{A}$ the activation temperature. Non-dimensioning is carried out with the use of the characteristics of a flame of reference, which has been chosen as the stoichiometric single-phase premixed flame. Namely, these characteristics are: (a) the stoichiometric mass fractions denoted by $Y_{i, u}^{*}$ for species $i$ [ $i=F, O$ and $p$, for fuel, oxygen and products, respectively]; (b) the adiabatic flame temperature $T_{b}^{*} ;$ (c) the corresponding thermal diffusivity in the burnt gases $D_{t h, b}^{*}=\lambda\left(T_{b}^{*}\right) /\left(\rho_{b}^{*} C_{p}\right) ;(d)$ the laminar velocity of the stoichiometric flame $U_{L}^{*}$.

Within the framework of a single step chemical scheme, note that $T_{b}^{*}$, the adiabatic flame temperature, allows us to define $Q^{*}$, the heat of reaction associated to expression (A.1) at stoichiometry, as follows

$$
Q^{*}=C_{p}\left(T_{b}^{*}-T_{u}\right) v_{F} M_{F} /\left(Y_{F}\right)_{u}^{*}
$$


As a matter of fact, the non-dimensioning we use resorts to the following theoretical expression of the laminar velocity, as derived by Joulin and Mitani [38] (see also Garcia-Ybarra et al. [39]).

$$
\left(U_{L}^{*}\right)^{2}=\frac{4}{Z e^{3}}\left(\frac{\rho_{b}^{*}}{\rho_{u}^{*}}\right)^{2} \frac{\lambda_{b}}{\rho_{b}^{*} C_{p}}\left[\left(\rho_{b}^{*}\right)^{2} v_{F} M_{F} Y_{o, u}^{*}\right] \exp \left(-T_{A} / T_{b}^{*}\right)
$$

Note that superscript "*" denotes a quantity related to the stoichiometric composition, while subscript "u" [resp. "b"] is associated with gaseous fresh [resp. burnt] mixture. As classically, $D_{t h, b}^{*}$ and $U_{L}^{*}$ allow us to define two scales for time and length, the so-called transit time and flame thickness, as follows

$$
\tau_{R D}^{*}=D_{t h, b}^{*} /\left(U_{L}^{*}\right)^{2} \quad l_{R D}^{*}=D_{t h, b}^{*} / U_{L}^{*}
$$

Hence, for the sake of simplifying the notations, $\left\{t, \mathrm{x}, \overline{\mathrm{V}}, \rho, D_{t h}, D_{i}, F\left(\varphi_{u}\right)\right\}$ stand hereinafter for

$$
\left\{t / \tau_{R D}^{*}, \mathrm{x} / l_{R D}^{*}, \overline{\mathrm{V}} / U_{L}^{*}, \rho / \rho_{b}^{*}, D_{t h} / D_{t h, b}^{*}, D_{i} / D_{t h, b}^{*}, Q / Q^{*}\right\}
$$

Furthermore, temperature and species mass fractions are made dimensionless as follows

$$
\theta=\left(T-T_{u}\right) /\left(T_{b}^{*}-T_{u}\right) ; \psi_{i}=Y_{i} / Y_{i, u}^{*} \quad(i=F \text { for the alkane fuel and } i=O \text { for oxygen })
$$

We are now able to write Equ. (A.1-3) in non-dimensional form as

$$
\begin{aligned}
& \frac{\partial \theta}{\partial t}+\bar{V} \cdot \bar{\nabla} \theta=\frac{1}{\rho} \operatorname{div}\left(\rho D_{t h} \bar{\nabla} \theta\right)+F\left(\varphi_{u}\right) W\left(\rho, \psi_{i}, \theta\right) \\
& \frac{\partial \psi_{i}}{\partial t}+\bar{V} \cdot \bar{\nabla} \psi_{i}=\frac{1}{\rho} \operatorname{div}\left(\rho D_{i} \bar{\nabla} \psi_{i}\right)-\mu_{i} W\left(\rho, \psi_{i}, \theta\right)
\end{aligned}
$$

where

$$
W\left(\rho, \psi_{i}, \theta\right)=\frac{Z e^{3}}{4}\left(\frac{\rho_{u}^{*}}{\rho_{b}^{*}}\right)^{2} \psi_{F} \psi_{O}\left(\frac{\rho}{\rho_{b}^{*}}\right)^{3} \exp \left[Z e \frac{\theta-1}{1+\gamma(\theta-1)}\right]
$$

with the reduced coefficients

$$
\mu_{i}=v_{i} M_{i} /\left(v_{F} M_{F}\right)
$$

and the classical parameters, $Z e=T_{A}\left(T_{b}^{*}-T_{u}\right) /\left(T_{b}^{*}\right)^{2}$ and $\gamma=\left(T_{b}^{*}-T_{u}\right) / T_{b}^{*}$

It is worth noting that $F\left(\varphi_{u}\right)$, the reduced heat of reaction, is a quantity that is tuneable in function of the local composition of the fresh mixture, which is characterized by the field $F\left(\varphi_{u}\right)$ [see section 2] . This takes account of the heterogeneous composition of the mixture. Accordingly with the present nondimensioning, we have $F\left(\varphi_{u}=1\right)=1$.

Finally, we assume that all Lewis numbers $\left[L e_{i}=D_{t h} / D_{i}\right]$ are constant (i.e. the ratio of thermal diffusivity to molecular diffusivity is supposed constant for each species $I$; they are taken equal to $L e_{F}=1.8$ for the fuel and $L e_{O}=0.9$ for oxygen. The same hypothesis is set on the Prandtl number [ $\operatorname{Pr}=v / D_{\text {th }}$ is supposed constant]. So that, we obtain the following system of governing equations, that reads

$$
\begin{aligned}
& \frac{\partial \theta}{\partial t}+\bar{V} \cdot \bar{\nabla} \theta=\frac{1}{\rho} \operatorname{div}\left(\rho D_{t h} \bar{\nabla} \theta\right)+F\left(\varphi_{u}\right) W\left(\rho, \psi_{i}, \theta\right) \\
& \frac{\partial \psi_{i}}{\partial t}+\bar{V} \cdot \bar{\nabla} \psi_{i}=\frac{1}{L e_{i}} \frac{1}{\rho} \operatorname{div}\left(\rho D_{t h} \bar{\nabla} \psi_{i}\right)-\mu_{i} W\left(\rho, \psi_{i}, \theta\right)
\end{aligned}
$$

coupled with the Navier-Stokes equations 
where $P$ stands for pressure.

$$
\begin{aligned}
& \frac{\partial \rho}{\partial t}+\operatorname{div}(\rho \bar{V})=0 \\
& \frac{\partial \bar{V}}{\partial t}+\overline{\overline{\nabla V}} \bar{V}=\operatorname{Pr} \frac{1}{\rho} \operatorname{div}\left\{\rho D_{t h}\left[\overline{\overline{\nabla V}}+(\overline{\overline{\nabla V}})^{T}\right]\right\}-\frac{1}{\rho} \bar{\nabla} P
\end{aligned}
$$

System (A.14-17) has been solved numerically with a mixed numerical method that takes account of the y-periodicity, for which Fourier spectral methods is used. As for the x-direction, the numerical approach resorts to finite differences. A particular treatment for computing pressure has been implemented [32] and continuously improved [40], since the present isobaric combustion is characterized by low Mach number flows. As surface tension is neglected, the numerical approach can consider a single-fluid flow with very high variations of density, from the volumetric mass of a hot gas to that of a liquid fuel. In such a way, inertia of the dense phase is easily accounted for. To confine the liquid fuel into the droplets, diffusivity is frozen at low temperature [1]. Typical discretization is 3072 nodes in the (propagation) x-direction and 256 Fourier modes in the (transverse) y-direction. More precisely, about 15 nodes are at least present along a droplet radius. To check the numerical precision, this discretization has been doubled; no change in the observed features has been noticed.

The limitation of the numerical approach is that no immediate coupling with sound can be envisaged, since acoustic filtering is associated to low Mach number flow models, as well as highly compressible flow (or high speed combustion) cannot be considered. Another limitation is related to the absence of surface tension: if the length scale of the flow shears is smaller than the droplet size, freezing the diffusivity will be insufficient to confine the fuel inside the droplets.

\section{Appendix B}

This addendum is devoted to answer a practical question: is it possible to change $\varphi_{G}$ without drastically affecting the characteristic quantities used for the non-dimensional stage that has been described in Appendix A? This point is an important issue in terms of interpreting our results with respect to laboratory experiments.

Our numerical experiments have considered spray-flames, which are determined by the set of parameters that characterizes the initial mixture: pressure $P$, temperature $T_{u}$, chemical composition (i.e. $\varphi_{G}$ and $\varphi_{T}$ given) and droplet geometry (i.e. $S$ given). Evidently, such a spray-flame cannot generally exist from the experimental point of view, since $P, T_{u}$ and $\varphi_{G}$ are not independent. These quantities are linked by the Clausius-Clapeyron formula on saturated vapour. 
Furthermore, as flame of reference in the non-dimensional process, we have used the stoichiometric single-phase flame defined by the set $\left\{P, T_{u}, \varphi_{G}=\varphi_{T}=1\right\}$. Obviously, the latter only exists if the pair $\left\{P, T_{u}\right\}$ belongs to a certain domain, which is delimited by the conditions of saturated vapour.

Hence, instead of considering the spray-flame determined by $\left\{\varphi_{G}, \varphi_{T}, s\right\}$ for any $\left\{P, T_{u}\right\}$, the experimentalist has to perform the experiment for $\left\{P, T_{u}, \varphi_{G}\right\}$ satisfying the conditions of saturated vapour. For example, we can investigate two opposite cases: (a) we fix $T_{u}$ and consider $P\left(\varphi_{G}\right)$, or (b) we fix $P$ and consider $T_{u}\left(\varphi_{G}\right)$. Both strategies are studied below, for the range $0.1 \leq \varphi_{G} \leq 1$, discarding the limit case $\varphi_{G}=0$.

(a) we first consider that all spray-flames have the same initial temperature. Then, $p_{F}$, the saturated vapour pressure of fuel, is given. Therefore, $\varphi_{G}$ is experimentally controlled by the amount of air added to the mixture that surrounds the droplets. We hence obtain the pressure of the experiment as

$$
P\left(\varphi_{G}\right)=p_{F}+p_{O_{2}}\left(1+v_{N_{2}} / v_{O_{2}}\right)=p_{F}\left[1+\left(v_{O_{2}}+v_{N_{2}}\right) /\left(v_{F} \varphi_{G}\right)\right]
$$

To fix the idea, $P$ reads for isooctane

$$
P\left(\varphi_{G}\right)=p_{F}\left[1+60 / \varphi_{G}\right] \approx 60 p_{F} / \varphi_{G}
$$

Expressions (B.1-2) recall us that -for heavy alkanes- the total pressure is essentially determined by the amount of air. If we consider the range of variation $0.1 \leq \varphi_{G} \leq 1$, expression (B.2) indicates that total pressure will vary by a factor 10 . Although flame speed is known to vary mildly with pressure, this manner of monitoring $\varphi_{G}$ is not satisfactory, since flame thickness varies as $P^{-1}$. Therefore, using $\hat{P}$, a certain pressure value independent of $\varphi_{G}$, instead of $P\left(\varphi_{G}\right)$, introduces a huge error in assessing the length scale. We must turn to another manner of controlling $\varphi_{G}$.

(b) we now question the role of $T_{u}\left(\varphi_{G}\right)$, the fresh mixture temperature, which allows the experimentalists to monitor the fuel saturated vapour pressure for a given total pressure (i.e. a given amount of air, essentially). In combustion, it is well-known that the most sensitive quantity to temperature is the reaction rate. More precisely, it is known as an exponential function of the burnt gas temperature, which depends linearly on $T_{u}$ [as recalled in Eq.(3)]. In other words, the issue concerns the error committed by the fact of using $\hat{T}_{u}$, some constant value of the equilibrium temperature, instead of $T_{u}\left(\varphi_{G}\right)$. To fix the ideas about the effects of such a modification in reaction rate, let us estimate the error committed on the single-phase flame speed $U_{L}^{*}$ by the fact to use $\hat{T}_{u}$ instead of $T_{u}\left(\varphi_{G}\right)$. Eq. (A.5) recalls that reaction rate and flame speed squared are in direct link.

From the Clausius-Clapeyron formula, we relate $T_{u}$ and $\varphi_{G}$ as follows. 


$$
\frac{L}{R_{C} \hat{T}_{u}}\left[1-\frac{\hat{T}_{u}}{T_{u}}\right]=\ln \left(\frac{p_{F}}{\hat{p}_{F}}\right)=\ln \left(\frac{\varphi_{G}}{\hat{\varphi}_{G}}\right)
$$

where $p_{F}, L$ and $R_{C}$ are the saturated vapour pressure of fuel, the latent heat of fuel vaporization and the gas constant, respectively. Superscript "^" corresponds to the condition of saturated vapour for an intermediate value of $\varphi_{G}$, say $\hat{\varphi}_{G}=0.3$. Now, from Eq. (A.4-5) and Eq. (B.3), we can derive the following relation between $U_{L}^{*}\left(T_{u}\left(\varphi_{G}\right)\right) / U_{L}^{*}\left(\hat{T}_{u}\right)$ and $\varphi_{G}$ as

$$
\begin{aligned}
& \frac{U_{L}^{*}\left(T_{u}\right)}{U_{L}^{*}\left(\hat{T}_{u}\right)} \approx \exp \left\{-\frac{Z e\left[\hat{T}_{u}-T_{u}\left(\varphi_{G}\right)\right]}{2 \hat{T}_{b}^{*}}\right\} \approx \exp \left\{N_{R V} \ln \left(\varphi_{G} / \hat{\varphi}_{G}\right)\right\}=\left(\varphi_{G} / \hat{\varphi}_{G}\right)^{N_{R V}} \\
& \text { where } \\
& \qquad N_{V R}=\frac{Z e}{2} \frac{R_{C} \hat{T}_{u}}{L} \frac{\hat{T}_{u}}{\hat{T}_{b}^{*}}
\end{aligned}
$$

Note that $N_{V R}$ is a number that takes account of vaporization and reaction. For the isooctane flame under standard conditions, $N_{V R}$ is as small as 0.03 . In consequence, a ratio of $\varphi_{G} / \hat{\varphi}_{G}=3$ is damped into $U_{L}^{*}\left(T_{u}\left(\varphi_{G}\right)\right) / U_{L}^{*}\left(\hat{T}_{u}\right)=1.034$ by expression (B.4). This corresponds to a relative error on the order of $3 \%$. In other words, interpreting our results as obtained with the same initial temperature introduces an error on the reaction rate largely within the error bars of the experiments, as well as within those of the numerical methods. 


\section{References}

[1] Nicoli C., Denet B., Haldenwang P., (2014) Lean Flame Dynamics Through a 2D Lattice of Alkane Droplets in Air, Combust. Sci. Technol., 186: 2, 103-119.

[2] Nicoli C., Haldenwang P., Denet B. (2014) Simple Chemistry model for heterogeneous alkane-air mixtures: from premixed to spray flame - under consideration for publication in Combust. Sci. Technol.

[3] Myers G.D., Lefebvre A.H. (1986) Flame propagation in heterogeneous mixtures of fuel drops and air, Combust. Flame, 66: 193-210.

[4] Cekalin, E.K., (1961) Propagation of Flame in Turbulent Flow of Two-Phase Fuel-Air Mixture, Proc. Combust. Inst. 8: 1125-1129.

[5-a] Mizutani Y. and Nakajima A., (1973a), Combustion of Fuel Vapor-Drop-Air Systems Part I: Open Burner Flames, Combust. Flame 21: 343-350.

[5-b] Mizutani Y. and Nakajima A., (1973b), Combustion of Fuel Vapor-Drop-Air Systems Part II: Spherical Flames in a Vessel, Combust. Flame 20: 351-357.

[6] Hayashi S. and Kumagai S., (1975) Flame Propagation in Fuel Droplet-Vapor-Air Mixtures, Proc. Combust. Inst. 15: 445-451.

[7] Hayashi S., Kumagai S., Sakai T., (1976) Propagation velocity and structure of flames in droplet vapor-air mixtures, Combust. Sci. Technol. 15: 169-177.

[8] Polymeropoulos C. E., Das S., (1975) The Effect of Droplet Size on the Burning Velocity of KeroseneAir Sprays, Combust. Flame 25: 247-257.

[9] Ballal D.R., Lefebvre A.H., (1981) Proc. Combust. Inst. 18: 321-328.

[10] Nomura H., Koyama M., Miyamoto H., Ujiie Y., Sato J., Kono M., Yoda S., (2000) Microgravity Experiments of Flame Propagation in Ethanol Droplet Vapour-Air Mixture, Proc. Combust. Inst., 28: 9991005.

[11] Bradley D., Lawes M., Liao S., Saat A., (2014) Laminar mass burning and entrainment velocities and flame instabilities of iso-octane, ethanol and hydrous ethanol/air aerosols,

Combust. Flame 161:6 1620-1632.

[12] Nassouri M., Chauveau C., Halter F., Gökalp I. (2013) Flame structure of ethanol-air premixed mixtures at high pressures in microgravity, Proc. ECM2013, Lund, Sweden.

[13] Atzler F., (1999) Fundamental studies of aerosol combustion, Ph.D. Thesis, School of Mechanical Engineering, University of Leeds. 
[14] Bradley D., Cresswell T.M., Puttock J.S., (2001) Flame Acceleration Due to Flame-Induced Instabilities in Large-Scale Explosions, Combust. Flame 124: 551-559.

[15] Karlin V., Sivashinsky G., (2007) Asymptotic modelling of self-acceleration of spherical flames, Proc. Combust. Inst. 31: 1023-1030

[16] Fursenko R. V., Pan K. L., Minaev S. S., (2008) Noise influence on pole solutions of the Sivashinsky equation for planar and outward propagating flames, Phys. Rev E 78: 056301

[17]D'Angelo Y., Joulin G., Boury G., (2000) On model evolution equations for the whole surface of threedimensional expanding wrinkled premixed flames, Comb. Theory and Modelling, 4:3 317-338.

[18] Denet B., Joulin G., (2011) Wrinkled flames and geometrical stretch, Phys. Rev E 84: 016315.

[19] Mikami M., Oyagi H., Kojima N., Wakashima Y., Kikuchi M., Yoda S.,

Microgravity experiments on flame spread along fuel-droplet arrays at high temperatures,

Combust. Flame, 146 (2006) 391-406.

[20] S.K. Aggarwal and W.A. Sirignano, Unsteady spray flame propagation in a closed volume, Combust. Flame 62 (1985) 69-84).

[21] I. Silverman JB Greenberg, Y. Tambour , Stoichiometry and polydisperse effects in premixed spray flames, Combust. Flame 93 (1993) (97-118)

[22] Suard, S., Haldenwang, P., Nicoli, C., (2004) Different spreading regimes of spray flames., C. R. Acad. Sci. Mécanique (Paris), 332:(5-6) 387-396.

[23] A.Neophytou, E. Mastorakos, Simulations of laminar flame propagation in droplet mists, Combust. Flame 156 (2009) 1627-1640

[24] Reveillon J., Vervisch L., (2005) Analysis of weakly turbulent dilute-spray flames and spray combustion regime, J. Fluid Mech. 537: 317-347.

[25] M. Kikuchi , Y. Wakashima, S. Yoda , M. Mikami, Numerical study on flame spread of an n-decane droplet array in different temperature environment under microgravity, Proceedings of the Combustion Institute , 30 (2005) 2001-2009

[26] Nicoli, C., Haldenwang, P., Suard, S., (2005) Analysis of pulsating spray flames propagating in lean two-phase mixtures with unity Lewis number, Combust. Flame, 143: 299-312.

[27] Nicoli, C., Haldenwang, P., Suard, S., (2007) Effects of substituting fuel spray for fuel gas on flame stability in lean premixtures. Combust. Flame, 149: 295-313. 
[28] Hanai H., Maruta K., Kobayashi H., Niioka T., (1998) Pulsating Flame Propagation of PMMA ParticleCloud in Microgravity, Proc. Combust. Inst., 27: 2675-2681

[29] Nicoli C., Haldenwang P., (2010) "Analysis of one-step chemistry models for flame propagation in various equivalence ratio premixtures of high alkane-air", SPEIC10: Towards Sustainable Combustion, Tenerife, Spain.

[30] Garrido-Lopez D., Sarkar S., (2005), Effect of imperfect premixing coupled with hydrodynamic instability on flame propagation, Proc. Combust. Inst., 30: 621-628.

[31] Fernandez-Tarrazo E., Sanchez A. L., Linan A., Williams F. A., (2006) A simple one-step chemistry model for partially premixed hydrocarbon combustion, Combust. Flame, 147: 32-38.

[32] Denet B., Haldenwang P., (1995) A numerical study of premixed flames Darrieus-Landau instability, Combust. Sci. Technol., 104: 143-167.

[33] Patel N. and Menon S., (2008) A computational fluid dynamics study of propane/air microflame stability in a heat recirculation reactor, Combust. Flame, 153: 228 - 269

[34] Lawes M., Saat A., (2011) Burning rates of turbulent iso-octane aerosol mixtures in spherical flame explosions, Proc. Combust. Inst. 33: 2047-2054.

[35] Borghi R., Champion M., (2000) Modélisation et Théorie des Flammes. Technip, Paris.

[36] Demoulin F. X., Borghi R., (2002) Modeling of Turbulent Spray Combustion with Application to Diesel like Experiment, Combust. Flame 129: 281-293.

[37] Yamashita H., Shimada M. and Takeno T., (1996), A numerical study on flame stability at the transition point of jet diffusion flames, Proc. Combust. Inst., 26: 27-34.

[38] Joulin G., Mitani J., (1981) Linear stability analysis of two-reactant flames, Combust. Flame, 40: 235246.

[39] Garcia-Ybarra P., Nicoli C., Clavin P., (1984) Soret and Dilution Effects on Premixed Flames, Combust. Sci. Technol., 42: 87-109.

[40] Garba A., Haldenwang P., (2013) A Helmholtz-Hodge projection method using an iterative gauge computation to solve the 3D Generalized Stokes Problem,

SIAM Journal on Scientific Computing, 35-3: A1560-A1583 